Nonlinear Processes in Geophysics (2001) 8: 69-94

Nonlinear Processes

in Geophysics

(C) European Geophysical Society 2001

\title{
Intergyre transport in a wind-driven, quasigeostrophic double gyre: An application of lobe dynamics
}

\author{
Chad Coulliette ${ }^{1, *}$ and Stephen Wiggins ${ }^{2}$ \\ ${ }^{1}$ Control and Dynamical Systems and Environmental Engineering Science, 107-81, Caltech, Pasadena, CA 91125 \\ ${ }^{2}$ Control and Dynamical Systems, 107-81, Caltech, Pasadena, CA 91125 \\ * This research was supported by ONR Grant No. N00014-97-1-0071
}

Received: 6 July 1999 - Accepted: 18 November 1999

\begin{abstract}
We study the flow obtained from a three-layer, eddy-resolving quasigeostrophic ocean circulation model subject to an applied wind stress curl. For this model we will consider transport between the northern and southern gyres separated by an eastward jet. We will focus on the use of techniques from dynamical systems theory, particularly lobe dynamics, in the forming of geometric structures that govern transport. By "govern", we mean they can be used to compute Lagrangian transport quantities, such as the flux across the jet. We will consider periodic, quasiperiodic, and chaotic velocity fields, and thus assess the effectiveness of dynamical systems techniques in flows with progressively more spatiotemporal complexity. The numerical methods necessary to implement the dynamical systems techniques and the significance of lobe dynamics as a signature of specific "events", such as rings pinching off from a meandering jet, are also discussed.
\end{abstract}

\section{Introduction}

In this paper we consider intergyre transport in the top layer of a wind-driven, three-layer, quasigeostrophic double-gyre ocean model. We use techniques from dynamical systems theory (invariant manifolds, lobe dynamics) to describe the flow structures associated with intergyre transport as well as to perform precise calculations of the intergyre flux. Dynamical systems techniques allow us to give a rigorous definition of the boundary between the southern and northern gyres. By "rigorous" we mean that at a given time, all fluid particles in the so constructed southern (resp., northern) gyre either make a clockwise (resp., counterclockwise) revolution around the southern gyre, or have earlier made such a revolution to arrive at their location at the given time. This boundary is constructed from pieces of two special curves; an unstable manifold emanating from the western boundary and a stable manifold emanating from the eastern boundary.

Correspondence to: C. Coulliette (chad@ caltech.edu)
These curves intersect to form regions called lobes, and it is only the fluid that is inside the lobes that can participate in intergyre transport. Hence, the area of the lobes can be directly related to the intergyre transport. Moreover, as these lobes are the sole mechanism for intergyre transport, their movement and geometrical shape give a complete description of the intergyre transport process. For example, we are able to give a detailed description of the "geometrical alleyways" followed by particle trajectories that start in the northern gyre, move downwards along the western boundary current, transfer to the southern gyre, make a clockwise journey around the southern gyre, moving upwards along the western boundary current, and then transferring back to the northern gyre. Moreover, since these stable and unstable manifolds are the mediators of intergyre transport, the role of eddies in intergyre transport can also be analyzed. In particular, we are able to study the formation and kinematics of ring structures and determine their role in intergyre transport.

The dynamical systems approach to Lagrangian transport has been applied to a variety of problems in fluid mechanics. Babiano et al. (1994) and Aref and El Naschie (1994) provide recent reviews. Dynamical systems techniques were first applied to Lagrangian transport in the context of twodimensional, time-periodic flows. In recent years these techniques have been extended to include flows having arbitrary time dependence, see Wiggins (1992), Malhotra and Wiggins (1998), and Haller and Poje (1998). One aspect of our study is to consider the effect of different types of temporal variability on transport. Accordingly, we compare transport in flow regimes exhibiting periodic, quasiperiodic, and chaotic time dependence. In this sense, our work is in the same spirit as recent work concerned with understanding the bifurcation structure of the wind-driven quasigeostrophic equations (Dijkstra and Katsman, 1997) and the wind-driven shallow water equations (Jiang et al., 1995). In recent years the dynamical systems approach has been extended to a number of geophysical fluid dynamics settings, for example, Pierrehumbert (1991a,b), Samelson (1992), Duan and Wiggins (1996). These early works mainly involved kinematically 
defined velocity fields. Some of the first attempts to treat dynamically evolving velocity fields were the works of delCastillo-Negrete and Morrison (1993) and Ngan and Shepherd (1997). The treatment of general dynamically evolving velocity fields became possible with the development of computational techniques to treat velocity fields which only had a numerical representation, i.e., which were the output of the numerical solution of a partial differential equation whose solution was a velocity field. Early work along these lines is Shariff et al. (1992), Duan and Wiggins (1997), and Miller et al. (1997). Recent work of this type in a geophysical fluid dynamics setting is that of Rogerson et al. (1999), which is concerned with fluid exchange across a barotropic meandering jet, and that of Poje and Haller (1999), which is focused on ring detachment. The numerical techniques developed in this paper allow us to treat transport in basin scale models, whereas the previous works were limited to transport issues associated with certain features in flows (e.g., meandering jets, flow around obstacles, ring detachment).

This work is organized as follows. In $\S 2$ we describe the model. In $\S 3$ we describe the numerical methods, for which there are two parts. One is concerned with the numerical solution of the quasigeostrophic equations, the next part is concerned with the numerical methods for studying the trajectories of the velocity field obtained from the numerical solution of the quasigeostrophic equations. In $\S 4$ we review the basic ideas from dynamical systems theory that are used and show how they apply in the context of the intergyre transport problem. In $\S 5$ we describe the results.

\section{Description of the model}

We use a three-layer eddy-resolving quasigeostrophic model (Rowley, 1996) which has its origins in the two-layer model developed by Holland (1978) and is similar to the later models of Cummins and Mysak (1988), and Lozier and Riser $(1989,1990)$. The model uses the quasigeostrophic approximation in the context of a $\beta$-plane $\left(f=f_{0}+\beta y\right)$ ocean modeled as three discrete isopycnal layers, each of mean layer thickness and constant density, as depicted in Fig. 1.

Following Rowley (1996), each layer $k$ of the model is of mean thickness $H_{k}$ and constant density $\rho_{k}$. The reduced gravity between layers $k$ and $k+1$ in the model is

$g_{k+1 / 2}^{\prime}=\frac{g\left(\rho_{k+1}-\rho_{k}\right)}{\rho_{0}}$,

where $g$ and $\rho_{0}$ are the gravitational constant and a reference density. The $\rho_{0}$ with the set of $H_{k}$ and $\rho_{k}$ define the mean stratification of the model ocean. Variations of the total depth $H_{0}$ are represented by $h_{B}(x, y)$, with the restriction under quasigeostrophy that $h_{B} \ll H_{N}$, for a model with $N$ layers.

We can write the governing $N$-layer quasigeostrophic
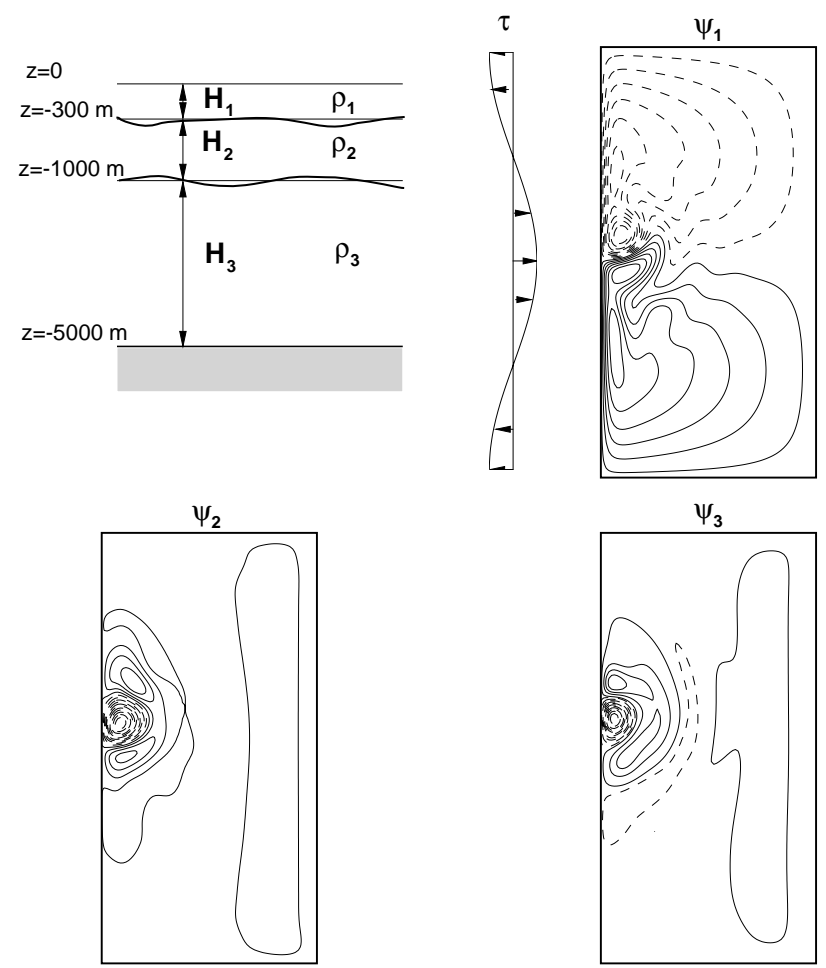

Fig. 1. In the upper left panel is a qualitatitve depiction of a three layer quasigeostrophic model. The other panels show streamfunction contours for each of the three layers which are typical for the parameter values chosen for this study. Note that the streamfunction contours are solid for positive values and dashed for negative values, which in the first layer correspond to an anticyclonic gyre and cyclonic gyre, respectively. A schematic profile of the prescribed wind stress $\tau$ is shown adjacent to the first layer.

equations on a $\beta$ plane (Holland, 1978),

$$
\begin{aligned}
& \frac{\partial}{\partial t} \nabla^{2} \psi_{k}=J\left(f+\nabla^{2} \psi_{k}, \psi_{k}\right) \\
& +\frac{f_{0}}{H_{k}}\left(w_{k-1 / 2}-w_{k+1 / 2}\right)+F_{k}+D_{k}, \\
& \frac{\partial}{\partial t} h_{k+1 / 2}=J\left(h_{k+1 / 2}, \psi_{k+1 / 2}\right)+w_{k+1 / 2}, \\
& h_{k+1 / 2}=\frac{f_{0}}{g_{k+1 / 2}^{\prime}}\left(\psi_{k+1}-\psi_{k}\right),
\end{aligned}
$$

where $\psi_{k}$ is the layer- $k$ quasigeostrophic streamfunction and $J$ is the Jacobian operator defined such that

$$
\begin{aligned}
J\left(\psi_{k}, A\right) & =\frac{\partial \psi_{k}}{\partial x} \frac{\partial A}{\partial y}-\frac{\partial \psi_{k}}{\partial y} \frac{\partial A}{\partial x} \\
& =u_{k} \frac{\partial A}{\partial x}+v_{k} \frac{\partial A}{\partial y} .
\end{aligned}
$$

The remaining variables in (2-4) are the interface height perturbations $h_{k+1 / 2}$, interface vertical velocities $w_{k+1 / 2}$, and the interface streamfunctions $\psi_{k+1 / 2}$, which are calculated 
as weighted averages of the corresponding layer function (Phillips, 1954), i.e.,

$$
\begin{aligned}
h_{k+1 / 2} & =\frac{H_{k} h_{k+1}+H_{k+1} h_{k}}{H_{k}+H_{k+1}}, \\
w_{k+1 / 2} & =\frac{H_{k} w_{k+1}+H_{k+1} w_{k}}{H_{k}+H_{k+1}}, \\
\psi_{k+1 / 2} & =\frac{H_{k} \psi_{k+1}+H_{k+1} \psi_{k}}{H_{k}+H_{k+1}},
\end{aligned}
$$

the force term $F_{k}$ and the dissipation term $D_{k}$. The forcing is through the wind stress $\tau=-\tau_{0} \cos \left(2 \pi y / L^{y}\right)$, so that $F_{k}$ is nonzero for $k=1$ only, when

$$
F_{1}=\frac{\operatorname{curl} \tau}{\rho_{0} H_{1}}=-\frac{2 \pi \tau_{0}}{\rho_{0} H_{1} L^{y}} \sin \left(\frac{2 \pi y}{L^{y}}\right) .
$$

Note that the imposed wind stress is sinusoidal and produces a double-gyre in the first layer, with an anticyclonic gyre in the southern part of the basin and a cyclonic gyre in the northern part of the basin (as shown in Fig. 1). In contrast, a more realistic forcing configuration is possible by mapping data for a given regional wind forcing from an acquisition grid to the model grid.

The dissipation is performed by lateral Laplacian friction, $D_{k}=v \nabla^{4} \psi_{k}$, which can include bottom drag, $D_{N}=$ $\nu \nabla^{4} \psi_{N}-c_{D} \nabla^{2} \psi_{N}$. The top and bottom boundary conditions enter through $w_{1 / 2}=0$, for a rigid lid, and $w_{N+1 / 2}=$ $D h_{B} / D t=J\left(\psi_{N}, h_{B}\right)$, for possible bottom topography.

Our goal is to study transport under different types of temporal variability. However, there are many parameters in this problem. In choosing the parameters we are guided by the work of Jiang et al. (1995) who isolated and presented results for several flow regimes for a wind-driven doublegyre, shallow water model. We have chosen basin dimensions and lateral dissipation coefficient identical to theirs. However, our resolution is significantly higher than that of Jiang et al. (1995), which will be discussed further in $\S 4.2$. The wind stress amplitude, $\tau_{0}$, was varied to obtain several flow regimes with different time dependencies, namely steady, periodic, quasiperiodic, and chaotic. The remaining model parameters, layer thicknesses, reduced gravity, mean state density, bottom friction coefficient, and Coriolis parameters, were chosen to be consistent with typical values used in three-layer quasigeostrophic models, such as that of Lozier and Riser (1989). The values of all the model parameters used throughout this study are given in Table 1 .

For a thorough discussion of the quasigeostrophic approximations, see Pedlosky (1977) or Cushman-Roisin (1994).

\section{Some key ideas from dynamical systems theory re- lated to transport}

In this subsection we introduce the ideas from dynamical systems theory that will be crucial for our analysis. Our discussion will be at an informal and intuitive level. Details of the mathematical background can be found in Malhotra and Wiggins (1998).

\begin{tabular}{|ll|}
\hline & \\
Basin dimensions & $L^{x}=1000 \mathrm{~km}$ \\
& $L \equiv\left(L^{x}+L^{y}\right) / 2=1500 \mathrm{~km}$ \\
Number of grid points & $N^{x}=81$ \\
& $N^{y}=161$ \\
Lateral dissipation coefficient & $v=300 \mathrm{~m}^{2} / \mathrm{s}$ \\
(Laplacian) & \\
Time step & $\Delta t_{\mathrm{PDE}}=2 \mathrm{~h}$ \\
Layer thickness & $H_{1}=300 \mathrm{~m}$ \\
& $H_{2}=700 \mathrm{~m}$ \\
Total ocean depth & $H_{3}=4000 \mathrm{~m}$ \\
Reduced gravity & $H_{0}=5000 \mathrm{~m}$ \\
& $g_{1}^{\prime}=0.03 \mathrm{~m} / \mathrm{s}^{2}$ \\
& $g_{2 \frac{1}{2}}^{\prime}=0.02 \mathrm{~m} / \mathrm{s}^{2}$ \\
Coriolis parameters & $f_{0}=9.3 \times 10^{-5} 1 / \mathrm{s}$ \\
Bottom friction & $\beta=2 \times 10^{-11} 1 / \mathrm{ms}$ \\
Mean state density & $C_{D}=1 \times 10^{-7} 1 / \mathrm{s}$ \\
Ekman number & $\rho_{0}=1.0 \mathrm{~g} / \mathrm{cm}^{3}$ \\
Rossby number & $E k \equiv v / \beta L^{3}=4.44 \times 10^{-6}$ \\
& $R o \equiv 2 \pi \tau_{0} / \rho_{0} \beta^{2} H L^{3}$ \\
\hline
\end{tabular}

Table 1. Model parameters.

\subsection{Hyperbolic trajectories or "moving saddle points"}

First, we want to generalize the notion of a saddle-type stagnation point of a steady flow to the setting of unsteady flows. In the case of a steady flow, the saddle point nature of a stagnation point is determined from the linearization of the flow about the saddle point. In that case the eigenvalues of the (constant) matrix associated with the linearization serve to characterize the saddle point nature of the stagnation point. In the case of aperiodically time dependent velocity fields, the saddle point nature of a time dependent trajectory is also determined from the linearization about the trajectory. However, in this case the eigenvalues of the (time dependent) matrix associated with the linearized velocity field cannot generally be used to determine the stability properties of the trajectory.

Consider the following two dimensional, unsteady velocity field

$\dot{\boldsymbol{x}}=\boldsymbol{v}(\boldsymbol{x}, t), \quad \boldsymbol{x} \in \mathbb{R}^{2}, \quad t \in \mathbb{R}$,

and let $\boldsymbol{x}=\boldsymbol{\gamma}(t)$ be a trajectory of (5). Then $\boldsymbol{\gamma}(t)$ is called a hyperbolic or saddle-type trajectory if the velocity field linearized about the trajectory:

$\dot{\xi}=\partial_{x} \boldsymbol{v}(\boldsymbol{\gamma}(t), t) \boldsymbol{\xi}, \quad \boldsymbol{\xi} \in \mathbb{R}^{2}$,

has two time-dependent, linearly independent solutions; one that grows unboundedly at an exponential rate as $t \rightarrow \infty$, 
which we refer to as $\varphi^{u}(t)$, and one that decays to zero at an exponential rate as $t \rightarrow \infty$, which we refer to as $\varphi^{s}(t)$. Hence, we can think of $\boldsymbol{\gamma}(t)$ as a "moving saddle point".

3.2 Stable and unstable manifolds of hyperbolic trajectories

With the notion of a hyperbolic trajectory defined, we can now define the stable and unstable manifolds associated with a hyperbolic trajectory. The term "manifold" is from dynamical systems theory, in the context of fluid mechanics it can be thought of as a distinguished material surface, whose nature we describe more fully below. Just as the notion of a hyperbolic trajectory follows from the linearized behavior near the trajectory, the stable and unstable manifolds associated with a hyperbolic trajectory also are related to the linearized behavior near the trajectory. In fact, we infer their existence based on the linearized behavior. So first we will describe the linearized behavior associated with the linearized velocity field.

Since (6) is linear, $\alpha \varphi^{u}(t)$, for all $\alpha \in \mathbf{R}$, is also a solution of (6) that grows unboundedly at an exponential rate as $t \rightarrow \infty$. At each instant in time, the set of points defined by $\alpha \varphi^{u}(t)$, for all $\alpha \in \mathbb{R}$, defines a one-dimensional subspace of $\mathbb{R}^{2}$, which we refer to as the unstable subspace, $\mathbf{E}^{u}(t)$, associated with the hyperbolic trajectory $\boldsymbol{\gamma}(t)$. As $t$ varies, this subspace may move. Clearly, it has the interpretation as the set of initial conditions, at time $t$, corresponding to trajectories that grow at an exponential rate as $t \rightarrow \infty$.

Similarly, $\alpha \varphi^{s}(t)$, for all $\alpha \in \mathbb{R}$, is also a solution of (6) that decays to zero at an exponential rate as $t \rightarrow \infty$. At each instant in time, the set of points defined by $\alpha \varphi^{s}(t)$, for all $\alpha \in \mathbb{R}$, defines a one-dimensional subspace of $\mathbb{R}^{2}$, which we refer to as the stable subspace, $\mathbf{E}^{s}(t)$, associated with the hyperbolic trajectory $\boldsymbol{\gamma}(t)$. As $t$ varies, this subspace may move, and it has the interpretation as the set of initial conditions, at time $t$, corresponding to trajectories that decay to zero at an exponential rate as $t \rightarrow \infty$.

One can view the stable and unstable manifold theorem for hyperbolic trajectories as saying that the behavior described above for the linearization of the velocity field about the hyperbolic trajectory $\boldsymbol{\gamma}(t)$ persists for the nonlinear velocity field (5). In particular, it states that there exists time varying curves, $\mathbf{W}^{s}(\boldsymbol{\gamma}(t))$ and $\mathbf{W}^{u}(\boldsymbol{\gamma}(t))$, that intersect in $\boldsymbol{\gamma}(t)$, and are tangent to $\mathbf{E}^{s}(t)$ and $\mathbf{E}^{u}(t)$, respectively, at $\boldsymbol{\gamma}(t)$. The curves $\mathbf{W}^{s}(\boldsymbol{\gamma}(t))$ and $\mathbf{W}^{u}(\boldsymbol{\gamma}(t))$ are referred to as the stable manifold and unstable manifold, respectively, associated with the hyperbolic trajectory $\boldsymbol{\gamma}(t)$, and they have the following properties.

Invariance. They are invariant curves, i.e., particle trajectories that start on the curves must stay on the curves for all time. This is the mathematical statement of the fact that they are material curves. Hence, they are barriers to transport in the sense that no particle trajectories can cross them.

Asymptotic Behavior. Particle trajectories that start on $\mathbf{W}^{s}(\boldsymbol{\gamma}(t))$ approach $\gamma(t)$ at an exponential rate as $t \rightarrow \infty$. Trajectories that start on $\mathbf{W}^{u}(\boldsymbol{\gamma}(t))$ approach $\boldsymbol{\gamma}(t)$ at an exponential rate as $t \rightarrow-\infty$. time $=\tau$

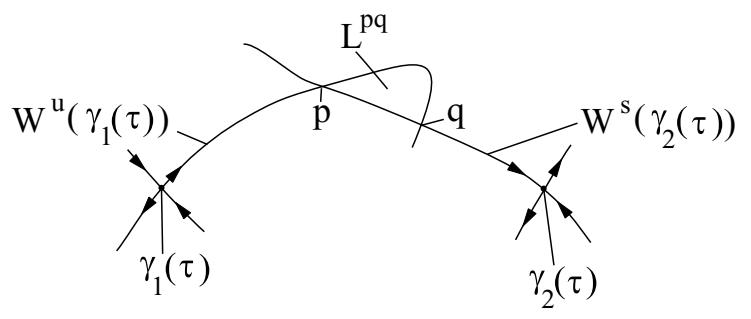

Fig. 2. A lobe, $L^{p q}$, at a fixed time $\tau$. Here the superscript " $p q$ " is used to explicitly denote the fact that this lobe is defined by segments of the stable and unstable manifolds that intersect at the primary intersection points $p$ and $q$. It will not be used in our later notation for lobes.

3.3 The implications of stable and unstable manifolds of hyperbolic trajectories for transport: Lobe dynamics

From the point of view of transport, the stable and unstable manifolds of hyperbolic trajectories are important because they form the "frontiers" between qualitatively different fluid particle trajectories. We now discuss the implications that stable and unstable manifolds of hyperbolic trajectories have for transport. In the following, $\boldsymbol{\gamma}_{i}(t)$ will denote a hyperbolic trajectory, with associated stable and unstable manifolds $\mathbf{W}^{s}\left(\boldsymbol{\gamma}_{i}(t)\right)$ and $\mathbf{W}^{u}\left(\boldsymbol{\gamma}_{i}(t)\right), i=1,2 .{ }^{1}$ We begin by defining the notion of a lobe.

A lobe is formed from intersections of stable and unstable manifolds of hyperbolic orbits in two dimensional flows and has played an important role in the study of transport in time periodic and quasiperiodic flows.

We now describe the generalization of "lobe dynamics" to two dimensional flows with aperiodic time dependence. First we define the notion of a primary intersection point of the stable and unstable manifolds of a hyperbolic trajectory.

At a fixed time $t=\tau$ consider a point $\boldsymbol{p} \in$ $\mathbf{W}^{s}\left(\boldsymbol{\gamma}_{1}(\tau)\right) \cap \mathbf{W}^{u}\left(\boldsymbol{\gamma}_{2}(\tau)\right)$. Let $\left[\boldsymbol{\gamma}_{1}(\tau), \boldsymbol{p}\right]$ denote the segment of $\mathbf{W}^{s}\left(\boldsymbol{\gamma}_{1}(\tau)\right)$ connecting $\boldsymbol{\gamma}_{1}(\tau)$ to $\boldsymbol{p}$ and let $\left[\boldsymbol{\gamma}_{2}(\tau), \boldsymbol{p}\right]$ denote the segment of $\mathbf{W}^{u}\left(\boldsymbol{\gamma}_{2}(\tau)\right)$ connecting $\boldsymbol{\gamma}_{2}(\tau)$ to $\boldsymbol{p}$. Then $\boldsymbol{p}$ is said to be a primary intersection point (pip) if $\left[\boldsymbol{\gamma}_{1}(\tau), \boldsymbol{p}\right]$ and $\left[\boldsymbol{\gamma}_{2}(\tau), \boldsymbol{p}\right]$ intersect only in $\boldsymbol{p}$.

With this definition in hand we can now define a lobe. Suppose $\boldsymbol{p}$ and $\boldsymbol{q}$ are two pips such that there are no other pips on the segments of $\mathbf{W}^{s}\left(\boldsymbol{\gamma}_{1}(\tau)\right)$ and $\mathbf{W}^{u}\left(\boldsymbol{\gamma}_{2}(\tau)\right)$ that connect $\boldsymbol{p}$ and $\boldsymbol{q}$. Then the region at the time $t=\tau$ bounded by the segments of $\mathbf{W}^{s}\left(\boldsymbol{\gamma}_{1}(\tau)\right)$ and $\mathbf{W}^{u}\left(\boldsymbol{\gamma}_{2}(\tau)\right)$ that connect $\boldsymbol{p}$ and $\boldsymbol{q}$ is called a lobe, which we denote by $L^{p q}$. The points $\boldsymbol{p}$ and $\boldsymbol{q}$ are referred to as the defining pips of the lobe, see Fig. 2.

First we write down two "rules" that must be satisfied by points on the stable and unstable manifolds of hyperbolic trajectories, which essentially follow from uniqueness of solu-

\footnotetext{
${ }^{1}$ With no loss of generality we could consider the case $\gamma_{1}(t)=$ $\gamma_{2}(t)$. This case is implicitly covered in our discussion, but it is not needed for the transport questions considered in this paper.
} 
tions and invariance of the manifolds.

Rule 1: Maintenance of Order Under Time Evolution. Since at any fixed time $t=\tau$ the curves $\mathbf{W}^{u}\left(\boldsymbol{\gamma}_{1}(\tau)\right)$ and $\mathbf{W}^{s}\left(\boldsymbol{\gamma}_{2}(\tau)\right)$ are one dimensional, points on them can be ordered. We define an ordering of points on $\mathbf{W}^{s}\left(\boldsymbol{\gamma}_{2}(\tau)\right)$ as follows. For any two points $\boldsymbol{q}_{\tau}, \overline{\boldsymbol{q}}_{\tau} \in \mathbf{W}^{s}\left(\boldsymbol{\gamma}_{2}(\tau)\right)$ we say that $\boldsymbol{q}_{\tau}<_{s} \overline{\boldsymbol{q}}_{\tau}$ if $\boldsymbol{q}_{\tau}$ is closer than $\overline{\boldsymbol{q}}_{\tau}$ to $\boldsymbol{\gamma}_{2}(\tau)$ in the sense of arc length along the curve $\mathbf{W}^{s}\left(\boldsymbol{\gamma}_{2}(\tau)\right)$. (A similar type of ordering can be defined for points on $\mathbf{W}^{u}\left(\boldsymbol{\gamma}_{1}(\tau)\right)$, but it will not be required in our construction.) Let $\boldsymbol{x}\left(t, \tau, \boldsymbol{q}_{\tau}\right) \equiv$ $\boldsymbol{q}_{\tau+t}, \boldsymbol{x}\left(t, \tau, \overline{\boldsymbol{q}}_{\tau}\right) \equiv \overline{\boldsymbol{q}}_{\tau+t}$ denote points at time $\tau+t$ that are the time evolution of the points $\boldsymbol{q}_{\tau}, \overline{\boldsymbol{q}}_{\tau}$. By invariance, these points are also in $\mathbf{W}^{s}\left(\boldsymbol{\gamma}_{2}(\tau+t)\right)$. Moreover, we have

$\boldsymbol{q}_{\tau+t}<_{s} \overline{\boldsymbol{q}}_{\tau+t}$.

This follows from uniqueness of solutions, otherwise there would be an intermediate time on which the trajectories passed through each other.

Rule 2: Invariance of Intersections. If the stable and unstable manifolds of a hyperbolic trajectory (or two different hyperbolic trajectories) intersect at a fixed time, then they intersect for all time. This simply follows from the fact that the manifolds are invariant for all time.

3.4 Lobe dynamics and flux: Quantification of finite time transport

Suppose $\boldsymbol{\gamma}_{1}(t)$ and $\boldsymbol{\gamma}_{2}(t)$ are hyperbolic trajectories. We assume that for some fixed time $\mathbf{W}^{u}\left(\boldsymbol{\gamma}_{1}(t)\right)$ and $\mathbf{W}^{s}\left(\boldsymbol{\gamma}_{2}(t)\right)$ intersect in $\tilde{N}$ points, where $\tilde{N}$ can be infinity. By invariance of the manifolds, if they intersect in $\tilde{N}$ points at one time, they must intersect in $\tilde{N}$ points for all other times. We also assume that the intersections are topologically transverse (i.e., heuristically, the manifolds pass through each other, and are not tangent at the intersection points of interest).

Let $\left\{t_{n}\right\}_{n=0}^{N}$ be a monotonically increasing sequence of times, and we could have $N=\infty$. These are the times at which we observe the flow. If $\boldsymbol{x}\left(t, t_{0}, \boldsymbol{x}_{0}\right)$ denotes the trajectory passing through the point $\boldsymbol{x}_{0}$ at $t=t_{0}$ then, for each $n \in\{0, \ldots, N\}$, we have the map

$\mathbf{f}_{n}: \boldsymbol{x}_{n} \mapsto \mathbf{f}_{n}\left(\boldsymbol{x}_{n}\right) \equiv \boldsymbol{x}\left(t_{n+1}, t_{n}, \boldsymbol{x}_{n}\right)=\boldsymbol{x}_{n+1}$,

which is just the mapping of points under the flow from time $t_{n}$ to $t_{n+1}$. (This notation makes certain formulae less cumbersome.) The inverse map is given by

$\mathbf{f}_{n}^{-1}: \boldsymbol{x}_{n+1} \mapsto \mathbf{f}_{n}^{-1}\left(\boldsymbol{x}_{n+1}\right) \equiv \boldsymbol{x}\left(t_{n}, t_{n+1}, \boldsymbol{x}_{n+1}\right)=\boldsymbol{x}_{n}$.

At time $t=t_{n}$ choose a point $\boldsymbol{q}_{n}$ on $\mathbf{W}^{u}\left(\boldsymbol{\gamma}_{1}\left(t_{n}\right)\right) \cap$ $\mathbf{W}^{s}\left(\boldsymbol{\gamma}_{2}\left(t_{n}\right)\right)$. Let $U\left[\boldsymbol{\gamma}_{1}\left(t_{n}\right), \boldsymbol{q}_{n}\right]$ denote the segment of $\mathbf{W}^{u}\left(\boldsymbol{\gamma}_{1}\left(t_{n}\right)\right)$ beginning at $\boldsymbol{\gamma}_{1}\left(t_{n}\right)$ and ending at $\boldsymbol{q}_{n}$ and let $S\left[\boldsymbol{\gamma}_{2}\left(t_{n}\right), \boldsymbol{q}_{n}\right]$ denote the segment of $\mathbf{W}^{s}\left(\boldsymbol{\gamma}_{2}\left(t_{n}\right)\right)$ beginning at $\boldsymbol{\gamma}_{2}\left(t_{n}\right)$ and ending at $\boldsymbol{q}_{n}$. The points in the sequence $\left\{\boldsymbol{q}_{n}\right\}$ are chosen such that

$\boldsymbol{q}_{n}<{ }_{s} \mathbf{f}_{n}^{-1}\left(\boldsymbol{q}_{n+1}\right), \quad \forall n \in \mathbb{Z}$,

and are referred to as boundary intersection points (bips). The sequence $\left\{\boldsymbol{q}_{n}\right\}$ can always be chosen to satisfy this constraint as a result of the fact that all points in
$\mathbf{W}^{u}\left(\boldsymbol{\gamma}_{1}(t)\right) \cap \mathbf{W}^{s}\left(\boldsymbol{\gamma}_{2}(t)\right)$ on a given time slice will have moved closer to $\mathbf{W}^{s}\left(\boldsymbol{\gamma}_{2}(t)\right.$ ) (closer in the sense of distance in arclength from $\boldsymbol{\gamma}_{2}(t)$ ) at any later time.

The importance of this condition will be apparent when we construct families of special lobes called "turnstiles" and describe their dynamical significance. This sequence of bips is used to construct a sequence of time-dependent boundaries and turnstiles.

Then $\mathcal{B}_{n} \equiv U\left[\boldsymbol{\gamma}_{1}\left(t_{n}\right), \boldsymbol{q}_{n}\right] \cup S\left[\boldsymbol{\gamma}_{2}\left(t_{n}\right), \boldsymbol{q}_{n}\right]$ is a curve at time $t_{n}$ joining $\mathbf{W}^{u}\left(\boldsymbol{\gamma}_{1}\left(t_{n}\right)\right)$ and $\mathbf{W}^{s}\left(\boldsymbol{\gamma}_{2}\left(t_{n}\right)\right)$. Locally, this curve separates the flow into two regions, which we denote by $R_{1}^{n}$ and $R_{2}^{n}$ (here the superscript on the regions indicates that they vary as $t_{n}$ varies; the superscript is the same as the subscript of $\left.t_{n}\right)$. We will be concerned with transport across this family of curves $\left\{\mathcal{B}_{n}\right\}_{n=0}^{N}$ at the sequence of times $\left\{t_{n}\right\}_{n=0}^{N}$ under the dynamics generated by the sequence of maps $\left\{\mathbf{f}_{n}\right\}_{n=0}^{N}$.

For an arbitrary time $t=t_{n-1}$ consider the point $\mathbf{f}_{n-1}^{-1}\left(\boldsymbol{q}_{n}\right) \in \mathbf{W}^{u}\left(\boldsymbol{\gamma}_{1}\left(t_{n-1}\right)\right) \cap \mathbf{W}^{s}\left(\boldsymbol{\gamma}_{2}\left(t_{n-1}\right)\right)$ (by the choice of the sequence $\left\{\boldsymbol{q}_{n}\right\}$, we have $\left.\boldsymbol{q}_{n-1}<_{s} \mathbf{f}_{n-1}^{-1}\left(\boldsymbol{q}_{n}\right)\right)$. Then there exists an odd number, $K_{n-1}$, of pips on $U\left[\mathbf{f}_{n-1}^{-1}\left(\boldsymbol{q}_{n}\right), \boldsymbol{q}_{n-1}\right]$ between $\mathbf{f}_{n-1}^{-1}\left(\boldsymbol{q}_{n}\right)$ and $\boldsymbol{q}_{n-1}$ (the odd number is due to the fact that the maps $f_{n}$ are orientation preserving due to uniqueness of trajectories passing through a given initial condition). These define $K_{n-1}+1$ lobes at time $t=t_{n-1}$; with $\left(K_{n-1}+1\right)-J_{n-1}$ in $R_{1}^{n-1}$, denoted by $L_{1,2}^{n-1}$, and $J_{n-1}$ lobes in $R_{2}^{n-1}$, denoted by $L_{2,1}^{n-1} .^{2}$ The lobes $L_{1,2}^{n-1} \cup L_{2,1}^{n-1}$ are called the turnstile lobes associated with the boundary $\mathcal{B}_{n-1}$ at the time $t=t_{n-1}$. The turnstile lobes are important because they mediate transport across the boundary $\mathcal{B}_{n-1}$.

The following theorem is the fundamental result, and is proved in Malhotra and Wiggins (1998).

\section{Theorem 3.1}

$\mathbf{f}_{n-1}\left(L_{1,2}^{n-1}\right) \subset R_{2}^{n}$,

$\mathbf{f}_{n-1}\left(L_{2,1}^{n-1}\right) \subset R_{1}^{n}$.

Moreover, the only points that move from $R_{1}^{n-1}$ (resp. $R_{2}^{n-1}$ ) into $R_{2}^{n}$ (resp. $R_{1}^{n}$ ) under the action of $\mathbf{f}_{n-1}$ by crossing $\mathcal{B}_{n-1}$ are those that are in $L_{1,2}^{n-1}$ (resp. $\left.L_{2,1}^{n-1}\right)$, see Fig. 3.

We remark that several "pathologies" for turnstile lobes are possible, e.g., turnstile lobes that intersect each other, and these can be treated in the aperiodic case exactly as described in Wiggins (1992).

Let $A$ denote an arbitrary region (but one sufficiently wellbehaved that its area is defined) in the flow. We denote the area of $A$ by $\mu(A)$. The instantaneous flux from $R_{1}^{n-1}$ into $R_{2}^{n}$ across $\mathcal{B}_{n}$ is given by

$\phi_{1,2}^{n-1}=\frac{1}{t_{n}-t_{n-1}} \mu\left(L_{1,2}^{n-1}\right)$.

\footnotetext{
${ }^{2}$ As we will see in the different flows considered in the following, the number of lobes in $R_{1}^{n}$ and $R_{2}^{n}$ may change as $n$ varies. This explains the need for the "time varying" integers $K_{n}$ and $J_{n}$ in the notation.
} 

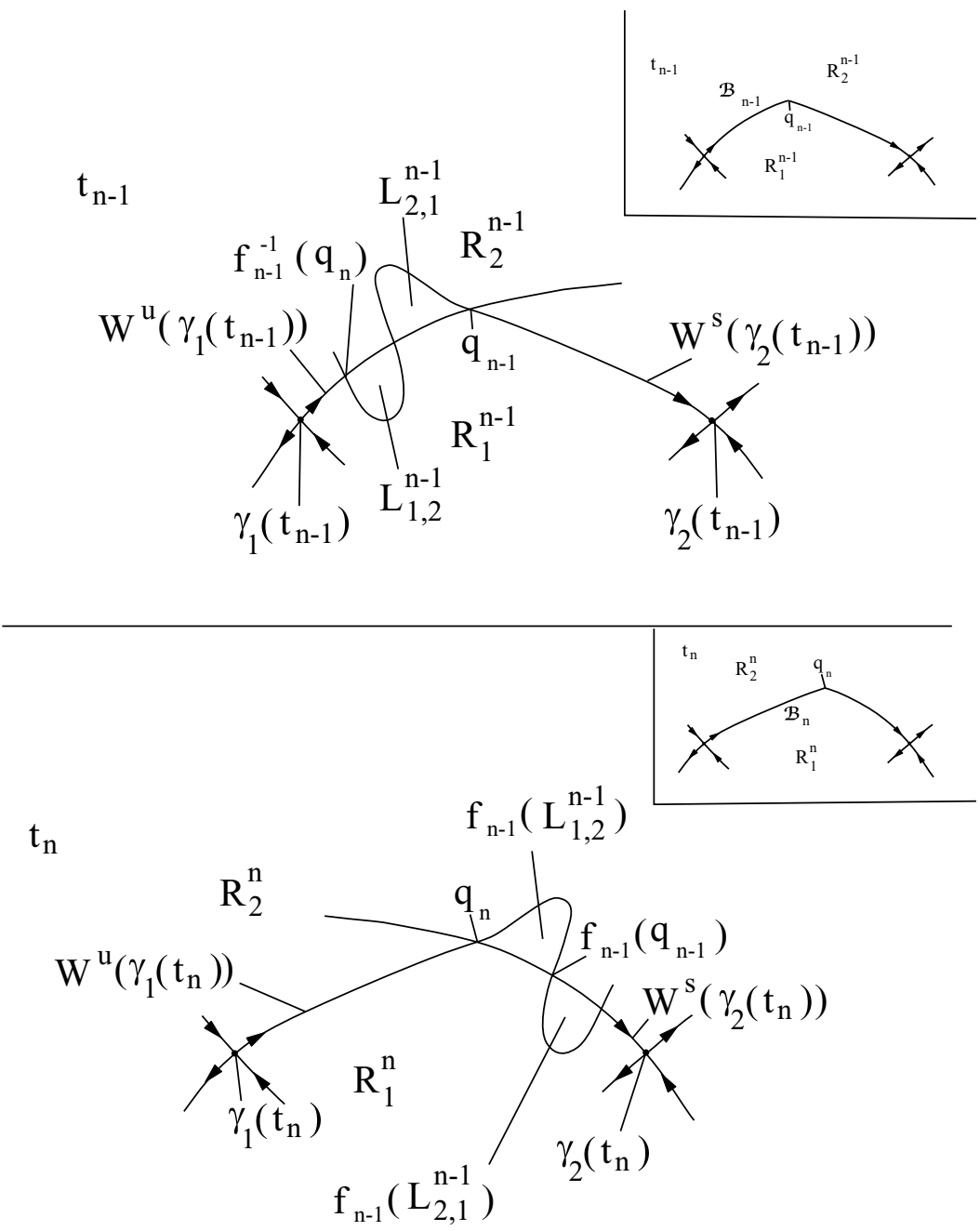

Fig. 3. The turnstile mechanism, illustrated for the case of two lobes per turnstile (i.e., $K_{n-1}=J_{n-1}=1$.) The insets in each panel show only the pieces of stable and unstable manifolds that make up the boundaries $\mathcal{B}_{n}$ at $t=t_{n}$ and $\mathcal{B}_{n-1}$ at $t=t_{n-1}$.

Similarly, the instantaneous flux from $R_{2}^{n-1}$ into $R_{1}^{n}$ across $\mathcal{B}_{n}$ is given by

$\phi_{2,1}^{n-1}=\frac{1}{t_{n}-t_{n-1}} \mu\left(L_{2,1}^{n-1}\right)$.

The weighted finite time average flux from $R_{1}^{0}$ into $R_{2}^{n}$ from time $t=0$ to $t=t_{n}$ is given by

$$
\begin{aligned}
\phi_{1,2} & =\frac{\left(t_{1}-t_{0}\right) \phi_{1,2}^{0}+\left(t_{2}-t_{1}\right) \phi_{1,2}^{1}+\cdots+\left(t_{n}-t_{n-1}\right) \phi_{1,2}^{n-1}}{\left(t_{1}-t_{0}\right)+\left(t_{2}-t_{1}\right)+\cdots+\left(t_{n}-t_{n-1}\right)}, \\
& =\frac{1}{t_{n}-t_{0}} \sum_{k=0}^{n-1} \mu\left(L_{1,2}^{k}\right) .
\end{aligned}
$$

Similarly, the weighted finite time average flux from $R_{2}^{0}$ into $R_{1}^{n}$ from time $t=0$ to $t=t_{n}$ is given by

$$
\begin{aligned}
\phi_{2,1} & =\frac{\left(t_{1}-t_{0}\right) \phi_{2,1}^{0}+\left(t_{2}-t_{1}\right) \phi_{2,1}^{1}+\cdots+\left(t_{n}-t_{n-1}\right) \phi_{2,1}^{n-1}}{\left(t_{1}-t_{0}\right)+\left(t_{2}-t_{1}\right)+\cdots+\left(t_{n}-t_{n-1}\right)} \\
& =\frac{1}{t_{n}-t_{0}} \sum_{k=0}^{n-1} \mu\left(L_{2,1}^{k}\right) .
\end{aligned}
$$

\subsection{Lobe dynamics and intergyre transport}

As a consequence of the double-gyre structure of the flow, there are two hyperbolic trajectories, one on the western boundary and one on the eastern boundary, whose unstable and stable manifolds, respectively, form the boundary between the southern and northern gyres. Moreover, transport between these gyres is completely governed by the evolution and geometry of these manifolds, which we now describe.

The hyperbolic trajectory on the western boundary is the (moving) point at which the flow along the western boundary converges from the southern and northern gyres and subsequently moves into the interior of the flow. Mathematically, we know that such a trajectory exists and that it has associated with it an unstable manifold that extends into the interior of the flow. Similarly, on the eastern boundary there exists a (moving) point at which the flow separates as it collides with the eastern boundary, resulting in some fluid moving northwards along the eastern boundary and some moving southward. This trajectory on the boundary has associated with it 
a stable manifold that extends into the interior of the flow.

The unstable manifold emanating from the western boundary may intersect the stable manifold emanating from the eastern boundary. An intersection point is primary (pip) if the segment of the unstable manifold from the western boundary to the intersection point does not intersect the segment of the stable manifold from the intersection point to the stable hyperbolic trajectory. All other intersection points are secondary (sips). It is important to distinguish between primary and secondary intersection points because it is the sequence of primary intersection points (pips) and the segments of unstable and stable manifolds between them that define the lobes. If the time dependence of the flow is periodic or quasiperiodic, then an infinite number of lobes are created. In the temporally chaotic cases we consider, numerical evidence shows that an arbitrarily large number of lobes exist.

Suppose that at some initial time, $t=t_{0}$, the stable and unstable manifolds intersect in a finite number of points as shown in Fig. 4. We stress here that the illustrations in this section are schematic in the sense that we show only enough detail of the manifolds to describe the ideas we are trying to convey. For example, in Fig. 4 we only show a relatively small piece of the unstable manifold in comparison with the length of the stable manifold shown. In practice one can only compute finite lengths of the manifolds even though in theory they are infinite in length.

At time $t=t_{0}$ we define a boundary between the southern and northern gyres by taking the pieces of the stable and unstable manifolds only up to some (arbitrary) pip labeled $a_{0}$ in Fig. 4 (the significance of the pip $b_{0}$ in this figure will be explained shortly). Once this boundary is chosen, we denote the resulting fluid in the southern gyre by the hatched region, see Fig. 4.

Now we consider the time evolution of these manifolds from $t=t_{0}$ to some later time $t=t_{n}$. Under time evolution segments of the unstable manifold lengthen, segments of the stable manifold shorten, and intersection points must remain intersection points (and obey the rules of time evolution described earlier). We illustrate the situation in Fig. 5. Hence we see that the boundary between the southern and northern gyres constructed at $t=t_{0}$ has deformed under time evolution.

We now ask the following question:

\section{How much fluid from the southern (resp. northern) gyre at time $t=t_{0}$ has moved into the northern (resp. southern) gyre at time $t=t_{n}$ ?}

The first thing we must do in order to answer this question is to describe what the boundary is at $t=t_{n}$ that separates the southern and northern gyres since the corresponding boundary defined earlier at $t=t_{0}$ has undergone deformations in the meantime. Hence at time $t=t_{n}$ we choose a new boundary between the southern and northern gyres. This boundary is formed by taking pieces of the stable and unstable manifolds only up to the pip labeled $b_{n}$, as shown in Fig. 5. Now the point $b_{n}$ is not arbitrary, but it is the evolution of the point $b_{0}$ at time $t_{0}$ to time $t_{n}$. With this choice for the boundary at $t_{n}$, it follows that the lobes between the points $b_{n}$ and $a_{n}$ are the time evolved lobes at time $t_{0}$ between the points $b_{0}$ and $a_{0}$. Note that $a_{0}, b_{0}, a_{n}$ and $b_{n}$ are all pips by definition, but also, $a_{0}$ in Fig. 4 and $b_{n}$ in Fig. 5 are bips, because they are the pips which determine the pieces of the stable and unstable manifolds that form the Lagrangian fluid boundary between the northern and southern gyres.

It is easily seen from Fig. 5 that the amount of fluid that has moved from the southern gyre at $t=t_{0}$ into the northern gyre at $t=t_{n}$ is the area of the hatched lobes between the points $b_{n}$ and $a_{n}$. Similarly, the amount of fluid that has moved from the northern gyre at $t=t_{0}$ into the southern gyre at $t=t_{n}$ is the area of the non-hatched lobes between the points $b_{n}$ and $a_{n}$.

It is possible to describe this procedure in a different way, but that gives the same meaning to intergyre transport.

At some time $t=t_{n}$ construct a boundary between the southern and northern gyres by choosing an intersection point, say $b_{n}$ as shown in Fig. 5 .

Once this boundary is chosen consider a set of lobes that is defined by moving to the right along the stable manifold and and stopping at some other intersection point, denoted $a_{n}$ in Fig. 5 .

The area of these lobes is then the amount of fluid that has crossed the boundary between the southern and northern gyres at $t=t_{n}$ from an earlier time $t_{0}$, where the boundary at $t_{0}$ was formed by taking the pieces of the stable and unstable manifolds only up to the point of intersection labelled $a_{0}$ in Fig. 4, where $a_{0}$ is the point at $t=t_{0}$ that evolves to $a_{n}$ at $t=t_{n}$.

In general, the areas of these two sets of lobes (i.e., the hatched lobes and the non-hatched lobes) are not the same, even though the flow is incompressible. This is because the boundary across which we are measuring transport may be moving in the sense that the boundary between the the southern and northern gyres at $t=t_{0}$ may not be the same as the boundary at $t=t_{n}$. This raises another transport question:

\section{How much fluid from the southern (resp. northern) gyre at time $t=t_{0}$ has crossed the boundary that was defined at $t_{0}$ ?}

The answer to this question is most easily seen by superimposing Figs. 4 and 5, as shown in Fig. 6. We see from this figure that the movement of fluid in the southern (resp. northern) gyre is due to two effects: 1) the change in the definition of the boundaries between the southern and northern gyres between $t=t_{0}$ and $t=t_{n}$ and 2) the movement of the lobes. We call transport due to the boundary movement reversible transport and transport due the lobes irreversible transport. The term irreversible transport derives from the fact that the fluid inside the lobes may travel arbitrarily far throughout the flow. This is due to the fact that the two primary intersection points that define the lobe get closer and closer as time increases since they both must approach the hyperbolic trajectory on the eastern boundary. Since the flow is incompressible the area of a lobe must remain the same for 


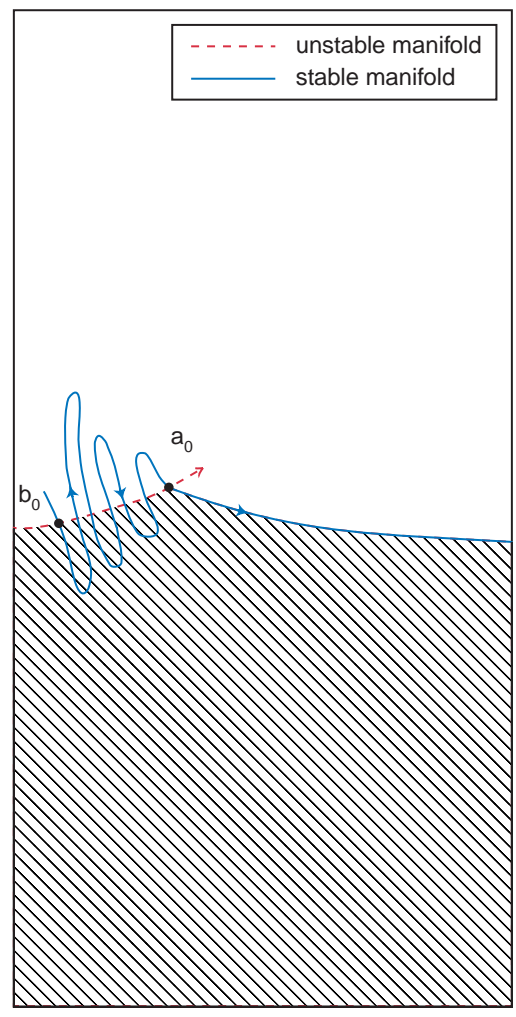

Fig. 4. Pieces of the unstable manifold of the hyperbolic trajectory on the western boundary and the stable manifold of the hyperbolic trajectory on the eastern boundary at time $t=t_{0}$.

all time. Hence the lobe must develop into a long, filamentary structure that winds throughout the flow.

It is possible to quantify the relative amounts of reversible and irreversible transport. The (signed) ${ }^{3}$ area between the boundaries at $t=t_{0}$ and $t=t_{n}$ is equal to the difference between the areas of the lobes in the southern gyre and the lobes in the northern gyre $t=t_{n}$. This computation is an elementary application of incompressibility of the flow, which we do not perform here but, rather, we explain the idea in Fig. 7. In panel a) of the figure we show the boundary at $t=t_{0}$ and the boundary at $t=t_{n}$, where along with the latter we also show the lobes that have crossed this boundary from the earlier time slice $t=t_{0}$. In panel b) we show the boundary between the southern and northern gyre at $t=t_{0}$ and the boundary between the southern and northern gyre at $t=t_{n}$. Here we show these two boundaries not intersecting. However, it is possible that they may intersect, but that does not have any effect on our conclusion (but it is the reason that we emphasized that we are computing signed areas). In panel c) we show the lobes in the southern and northern gyres along the boundary between the southern and northern gyres at $t=t_{n}$ (where we are only showing the segment of the stable manifold that makes up the boundary). The signed

\footnotetext{
${ }^{3}$ When the area is computed as a line integral around a boundary we take the positive sign when traversing the curve in a counterclockwise sense.
}

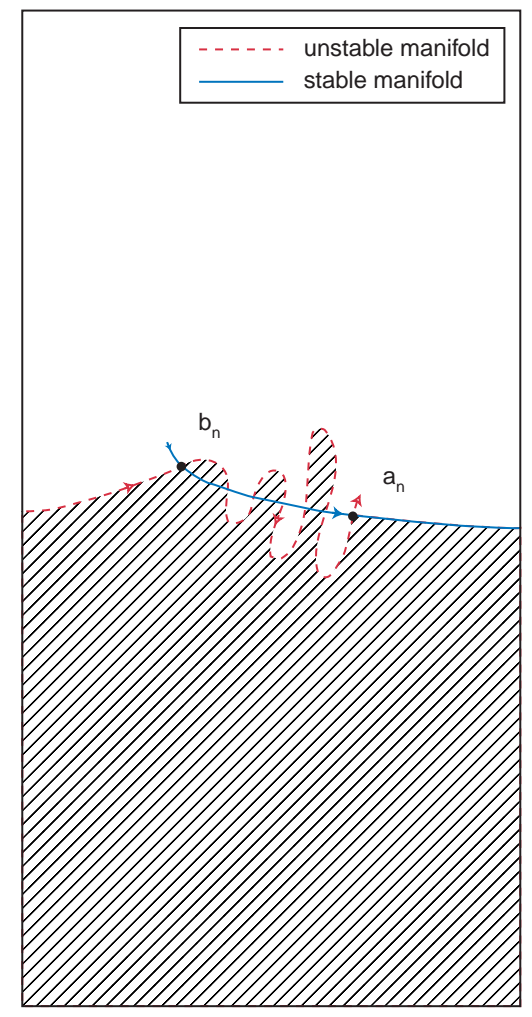

Fig. 5. The time evolution of the pieces of the unstable manifold of the hyperbolic trajectory on the western boundary and the stable manifold of the hyperbolic trajectory on the eastern boundary from time $t=t_{0}$ to $t=t_{n}$.

area between the two curves in b) is equal to the area of the lobes in the southern gyre minus the area of the lobes in the northern gyre shown in c).

\section{Numerical methods}

The numerical methods necessary for this study are divided into four parts: the solution of the quasigeostrophic equation described previously, the integration of Lagrangian trajectories, the construction of invariant manifolds of hyperbolic trajectories, and the determination of pips and the resulting lobes.

\subsection{Model solution}

After using (3) and (4) to eliminate $w_{k+1 / 2}$ in (2), the set of layer equations can be written in matrix form as (Rowley, 1996),

$$
\frac{\partial}{\partial t}\left(\nabla^{2} \hat{\boldsymbol{\Psi}}-\mathbf{A} \hat{\boldsymbol{\Psi}}\right)=\hat{R}
$$

where $\hat{\boldsymbol{\Psi}}$ is now a column vector of the $\psi_{k}$, the source terms on the right hand side are in a corresponding vector form $\hat{R}$, and the matrix A contains the constant coefficients that 


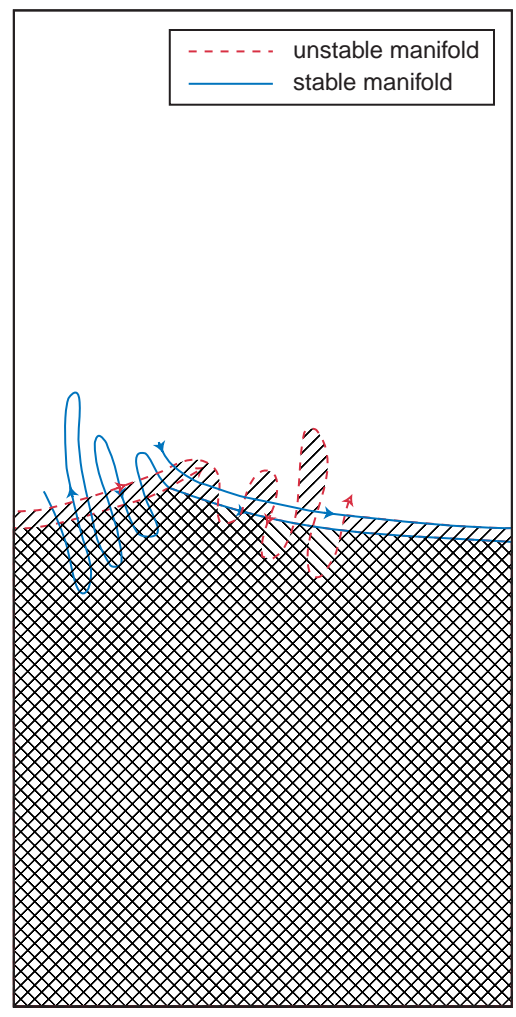

Fig. 6. Figures 4 and 5 superimposed.

couple the layer equations together. The elements of $\hat{R}$ are

$$
\begin{aligned}
\hat{R}_{k}= & J\left(f+\nabla^{2} \psi_{k}, \psi_{k}\right) \\
& +\frac{f_{0}^{2}}{g_{k-1 / 2}^{\prime} H_{k}} J\left(\psi_{k-1}-\psi_{k}, \psi_{k-1 / 2}\right) \\
& -\frac{f_{0}^{2}}{g_{k+1 / 2}^{\prime} H_{k}} J\left(\psi_{k}-\psi_{k+1}, \psi_{k+1 / 2}\right)+A_{H} \nabla^{4} \psi_{k} \\
& -\delta_{N, k} c_{D} \nabla^{2} \psi_{k}+\delta_{1, k} \frac{\operatorname{curl} \tau}{\rho_{0} H_{1}}-\delta_{N, k} J\left(\psi_{k}, \frac{f_{0} h_{B}}{H_{k}}\right) .
\end{aligned}
$$

The tridiagonal matrix $\mathbf{A}$ is given by

$$
\mathbf{A}=\left(\begin{array}{ccccc}
\alpha_{1,1} & \alpha_{1,2} & 0 & 0 & 0 \\
\alpha_{2,1} & \alpha_{2,2} & \alpha_{2,3} & 0 & 0 \\
0 & \alpha_{3,2} & \alpha_{3,3} & \alpha_{3,4} & 0 \\
\vdots & & & & \\
0 & 0 & \alpha_{N-1, N-2} & \alpha_{N-1, N-1} & \alpha_{N-1, N} \\
0 & 0 & 0 & \alpha_{N, N-1} & \alpha_{N, N}
\end{array}\right)
$$

which has nonzero elements

$$
\begin{aligned}
& \alpha_{k, k-1}=-\frac{f_{0}^{2}}{g_{k-1 / 2}^{\prime} H_{k}} \\
& \alpha_{k, k}=\frac{f_{0}^{2}}{g_{k-1 / 2}^{\prime} H_{k}}+\frac{f_{0}^{2}}{g_{k+1 / 2}^{\prime} H_{k}}
\end{aligned}
$$

$\alpha_{k, k+1}=-\frac{f_{0}^{2}}{g_{k+1 / 2}^{\prime} H_{k}}$.

The vertical boundary conditions (rigid lid, rigid bottom) are imposed through the $\alpha_{i j}$ by setting $g_{1 / 2}^{\prime}$ and $g_{N+1 / 2}^{\prime}$ to $\infty$. In practice, we non-dimensionalize (14) and solve the system by transforming to modal form, so that the layer equations are decoupled. This yields a transient solution for the streamfunction $\psi$ in each layer, which can be used directly for our analysis.

\subsection{Lagrangian trajectories}

For incompressible two-dimensional flow the velocity field is given in terms of the streamfunction $\psi(x, y, t)$, with $u(x, y, t)=-\partial \psi / \partial y$ and $v(x, y, t)=\partial \psi / \partial x$. Lagrangian trajectories of fluid particles satisfy

$$
\begin{aligned}
& \frac{d x}{d t}=u=-\frac{\partial \psi}{\partial y}(x, y, t), \\
& \frac{d y}{d t}=v=\frac{\partial \psi}{\partial x}(x, y, t) .
\end{aligned}
$$

Although the model of Rowley (1996) has the capability of non-rectangular boundaries, in this study the spatial domain is rectangular, defined by $0 \leq x \leq L^{x}$ and $0 \leq y \leq L^{y}$. The temporal domain is defined by $-T \leq t \leq T$, with the magnitude of $T$ determined by the particular case under consideration. For a periodic case, $T$ is simply the length of the period, but for more general time dependencies, $T$ is chosen to provide a large enough data set for analysis.

The numerical solution of the streamfunction discussed in $\S 4.1$ is known only at discrete temporal and spatial grid points, i.e. $\psi\left(x_{i}, y_{j}, t_{n}\right)$, where $i=1,2, \ldots, N^{x}, j=$ $1,2, \ldots, N^{y}$ and $n=1,2, \ldots, N_{t}$. The values of $N^{x}, N^{y}$, $L^{x}$ and $L^{y}$ used throughout this study are given in Table 1 and $N_{t}$ is given by $N_{t} \equiv T / \Delta T$, where $\Delta T$ is the temporal resolution to be discussed in more detail below. The spatial resolution is thus $\Delta x=\Delta y=L^{x} /\left(N^{x}-1\right)=$ $L^{y} /\left(N^{y}-1\right)=12.5 \mathrm{~km}$ throughout this study, which is selected so that the Munk layer, with length $\delta_{M}=(v / \beta)^{1 / 3}$, is described by two grid points. The motivation to resolve the Munk layer with at least two grid points is described by Meacham and Berloff (1997). The lateral boundary conditions are free-slip, as opposed to the partial-slip and noslip conditions used by Munk (1950), which means that the model's dissipative boundary layer is not a Munk layer in the strict sense. However, the free-slip boundary condition used in the model creates the necessary vorticity gradient for the establishment of a dissipative boundary layer, although weaker than the Munk layer. Thus, the Munk layer is a conservative quantity to use for resolution selection.

To integrate (15), we use an adaptive step-size RungeKutta-Fehlberg (RKF45) method, which substantially reduces the computational time needed by constant step size methods, such as the typical fourth-order Runge-Kutta (RK4) method. For the RK4 method, we found that a step size of $\Delta t_{\mathrm{RK} 4}=2 \mathrm{~h}$ is necessary for most of our test cases to avoid 


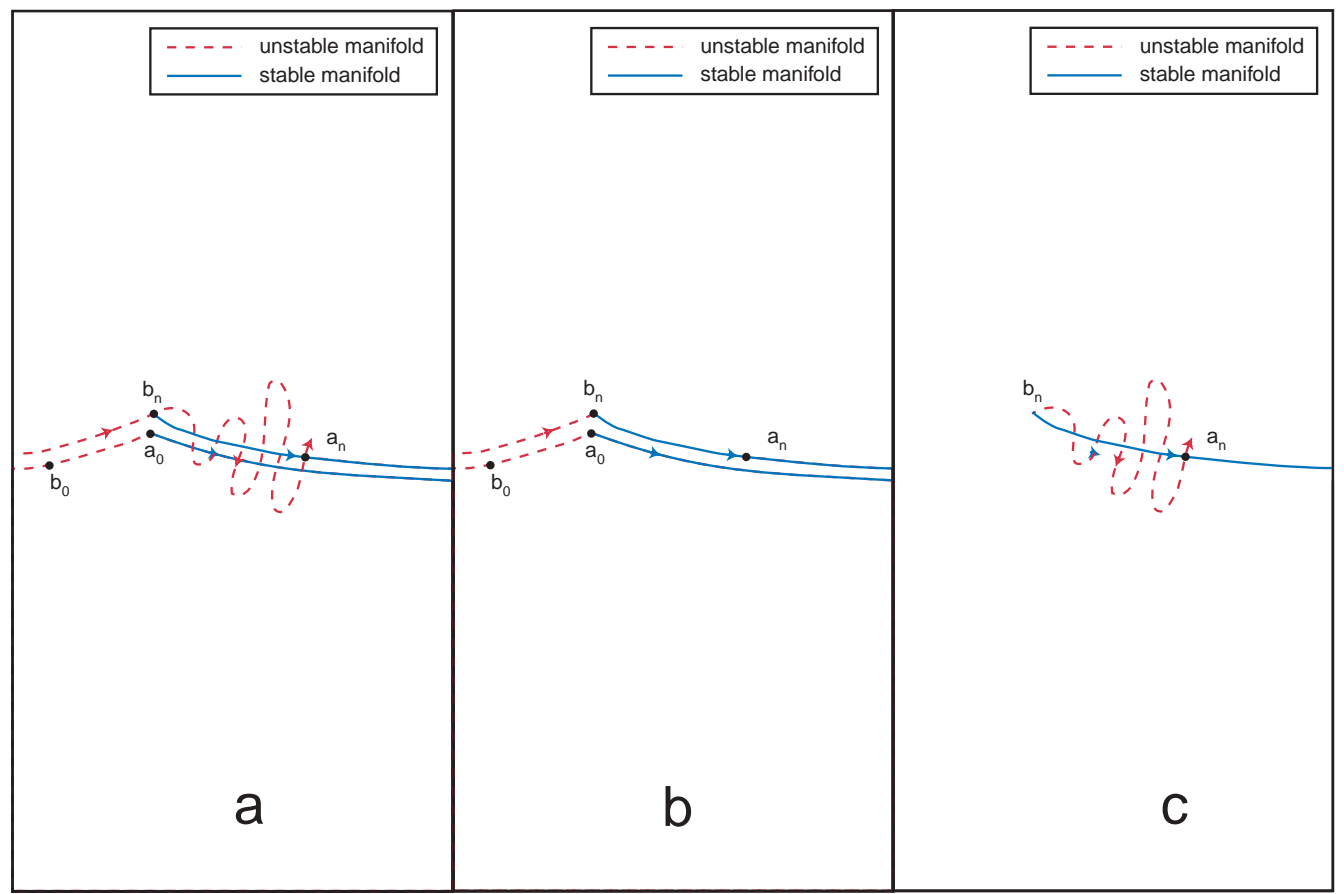

Fig. 7. (a) The boundary at $t=t_{0}$ (with no lobes shown) and the boundary at $t=t_{n}$ (with only the lobes shown that have crossed the boundary from the earlier time $t=t_{0}$ ). (b) The boundary between the southern and northern gyre at $t=t_{0}$ and the boundary between the southern and northern gyre at $t=t_{n}$. (c) The lobes in the southern and northern gyres along the boundary between the southern and northern gyres at $t=t_{n}$ (where we are only showing the segment of the stable manifold that makes up the boundary).

numerical instabilities (indicative of too large of an integration step). The step size of the RKF45 method is controlled by setting a tolerance $e_{t}$, a minimum step size $\Delta t_{\min }$, and a maximum step size $\Delta t_{\max }$, the values of which are shown in Table 2. We found that choosing $e_{t}=0.001$ yields a solution devoid of visible numerical instabilities, while simultaneously allowing the integration to efficiently proceed at a rate of $\Delta t_{\max }$ throughout most of the flow, only slowing to $\Delta t_{\min }$ in the jet and a few other nearby regions. For the aforementioned values of $\Delta t_{\mathrm{RK} 4}, e_{t}, \Delta t_{\min }$, and $\Delta t_{\max }$, the computational time required by RKF45 integration is approximately a factor of eight less than RK4 integration. In general, we found that the step size of the RKF45 method was inversely proportional to the norm of the velocity field, i.e., taking small steps when the speed of the flow was fast and large steps when the speed of the flow was slow. This suggests that a simpler adaptive step sizing method could be used by setting $\Delta t_{\mathrm{RK} 4}=\delta /|\boldsymbol{v}|$, where $\delta$ is an empirically found constant (Pozrikidis, 1992). The disadvantage of this type of adaptive method is choosing $\delta$ for each individual flow.

During integration, the velocity field is needed at an arbitrary point $(x, y, t)$, which requires interpolation in the three-dimensional space. Following the example of Miller et al. (1997), we divide the three-dimensional interpolation task into a two-dimensional spatial interpolation and a onedimensional temporal interpolation. To maintain the Hamiltonian structure of (15) and preserve area of the incompress- ible flow, it is necessary to interpolate the discrete streamfunction with a method that provides smoothness through the first derivative. We use local bicubic interpolation for the spatial interpolation of the streamfunction (Press et al., 1992), which directly yields values for $u$ and $v$. Bicubic interpolation requires values of $\psi, \partial \psi / \partial x, \partial \psi / \partial y$, and $\partial^{2} \psi / \partial x \partial y$ at the four discrete points nearest to $(x, y, t)$, i.e. $\left(x_{i ; i+1}, y_{j ; j+1}, t\right)$. The derivatives of $\psi$ are approximated by second-order numerical differentiation, thus requiring the value of $\psi$ at eight additional grid points adjacent to the aforementioned four points.

In order to obtain values for $u$ and $v$ at arbitrary $t$, temporal interpolation is performed using Lagrange polynomials. The motivation for using Lagrange polynomials, instead of a simpler method such as linear interpolation, is to decrease the temporal resolution $\Delta T$, thus reducing storage requirements and allowing a longer time evolution for data sets of general time dependence. The order of the Lagrange polynomial was varied from 1st degree through 6 th degree. Higher degree polynomials allowed larger $\Delta T$, thus reducing storage requirements, but computational time increases due to the increasing number of arithmetic operations. The computational time was found empirically to increase at the rate of $2.8 e^{0.337 N_{d}}$, where $N_{d}$ is the degree of the Lagrange polynomial. We chose to use 3rd degree polynomial interpolation for all of our tests, which offered the best compromise between storage requirements and computational time, decreasing the storage requirements by a factor of twelve over 
linear interpolation with $\Delta T=2 \mathrm{~h}$, while increasing the computational time by only a factor of two over linear interpolation.

Since 3rd degree polynomials require values for the interpolant at two temporal grid points before $t$ and two grid points after $t$, i.e. $t_{n-1}, t_{n}, t_{n+1}$, and $t_{n+2}$, the entire threedimensional interpolation scheme requires information from $(4+8) \times 4=48$ grid points. These grid points can be used in an interpolation scheme by performing bicubic spatial interpolation first or Lagrange polynomial temporal interpolation first. Our spatial-temporal interpolation method performs bicubic interpolation using twelve $\psi$ grid points in each of the four temporal data slices, then uses 3rd degree polynomials on the resulting four values of $u$ and $v$. Our temporalspatial interpolation method uses 3 rd degree polynomials to generate the necessary twelve grid points of $\psi$ at some arbitrary $t$, and then performs bicubic interpolation with those $\psi$ grid points to obtain $u$ and $v$. Although both the spatialtemporal and temporal-spatial methods use the same 48 grid points, efficiency and accuracy differ. Temporal-spatial interpolation is approximately $40 \%$ faster than spatial-temporal due to less arithmetic operations, but numerical oscillation occurs during computation of Lagrangian trajectories with the temporal-spatial method and not with the the spatialtemporal method. This is due to the inherent numerical error of the Lagrangian polynomials being magnified by the bicubic interpolation. We thus chose to use a spatial-temporal interpolation scheme for all of our results.

The temporal resolution $\Delta T$ is not equivalent to the time step used in solving the model $\Delta t_{\mathrm{PDE}}$, which is given in Table 1. $\Delta T$ may be chosen independently of $\Delta t_{\mathrm{PDE}}$, with the only restriction that $\Delta T$ must be an even multiple of $\Delta t_{\mathrm{PDE}}$. To choose $\Delta T$, we used the RK4 method with $\Delta t_{\mathrm{RK} 4}=2 \mathrm{~h}$ and 3rd degree polynomials, varying $\Delta T$ such that $\Delta T=$ $2,4, \ldots, 24 \mathrm{~h}$. The Lagrangian trajectories computed with $\Delta T=24 \mathrm{~h}$ were nearly indistinguishable from those computed with $\Delta T=2 \mathrm{~h}$. So, we chose to use $\Delta T=24 \mathrm{~h}$ for all of the computations in this study. A larger $\Delta T$ could perhaps have been used for interpolation purposes, but would be inconvenient for constructing the manifolds, as will be explained in the next section. To summarize, RKF45 integration using a spatial-temporal interpolation scheme with local bicubic splines and 3rd degree Lagrange polynomials is an excellent computational method for constructing Lagrangian trajectories, decreasing the computational time by a factor of four and simultaneously decreasing the storage requirements by a factor of twelve over a method with $\Delta T=\Delta t_{\mathrm{RK} 4}$, such as that used by Miller et al. (1997) and other workers that have followed their example.

\subsection{Hyperbolic trajectories and invariant manifolds}

Since the hyperbolic trajectories we are interested in for studying intergyre transport are located on either the western or eastern boundary, a rather straightforward method can be designed for finding and tracking the location of a hyperbolic trajectory, $\boldsymbol{\gamma}(t)$. As a hyperbolic trajectory travels ver-

\begin{tabular}{|ll|}
\hline RKF45 tolerance & $e_{t}=0.001$ \\
Minimum step size & $\Delta t_{\min }=1 \mathrm{~h}$ \\
Maximum step size & $\Delta t_{\max }=24 \mathrm{~h}$ \\
Temporal resolution & $\Delta T=24 \mathrm{~h}$ \\
\end{tabular}

Table 2. Temporal integration constants used in all of the results.

tically up and down either the western or eastern boundary, there will be a corresponding instantaneous stagnation point, $\boldsymbol{\gamma}^{s p}(t)$, which is also traveling up and down. There is a relationship between the range of travel of the hyperbolic trajectory and the range of travel of the stagnation point: across a sufficiently long time span, the range of travel of the hyperbolic trajectory will always be within the range of travel of the stagnation point. This relationship between the stagnation point and the hyperbolic trajectory is further explained and proven by Ide and Wiggins (2000). Thus, if we know the bounds on the range of travel of the stagnation point, then we also know the bounds on the range of travel of the hyperbolic trajectory. So, we begin by first devising and employing a method for finding the stagnation point. We estimate the location of the stagnation point $\gamma^{s p}(\tau)$ at a time $t=\tau$, either by visual inspection of the streamfunction contours or from a known location of the stagnation point at some previous time $t=\tau-\Delta t$, i.e. $\gamma^{s p}(\tau-\Delta t)$. We then initialize (15) with $x=\gamma_{x}^{s p}$ and $y=\gamma_{y}^{s p} \pm \delta_{y}$ at $t=\tau$, where $\delta_{y}$ is chosen large enough such that $\gamma_{y}^{s p}(\tau-\Delta t)-\delta_{y}<\gamma_{y}^{s p}(\tau)<$ $\gamma_{y}^{s p}(\tau-\Delta t)+\delta_{y}$ is expected, and then integrate each resulting pair of (15) through $\Delta t$. In practice, $\gamma_{x}^{s p}$ is not set exactly equal to the $x$-coordinate of the boundary, but rather is set to position that is a small distance from the boundary. The integration is performed by a constant step size integrator, such as RK4, and $\Delta t$ is a small value. In our case we chose $\Delta t= \pm 1 \mathrm{~h}$, where $\Delta t>0$ for hyperbolic trajectories on the western boundary and $\Delta t<0$ on the eastern boundary. It should be pointed out that the sign of $\Delta t$ is indirectly a result of which boundary the hyperbolic trajectory resides; directly, it is determined by the objective to compute either the unstable or stable manifold, being positive for the former and negative for the latter. In either case, if both trajectories resulting from the integration of the pair of (15) move toward the estimate $\gamma_{y}^{s p}(\tau)$, then we have two points which straddle the stagnation point. If we have not straddled the stagnation point, then we may either try to improve our guess for the present location of the stagnation point, or increase $\delta_{y}$ and check again if the stagnation point is straddled. Once we have straddled the stagnation point, then we proceed by letting $\left(\alpha_{x}^{s p}, \alpha_{y}^{s p}\right)=\left(\gamma_{x}^{s p}, \gamma_{y}^{s p}(\tau)-\delta_{y}\right)$ and $\left(\beta_{x}^{s p}, \beta_{y}^{s p}\right)=\left(\gamma_{x}^{s p}, \gamma_{y}^{s p}(\tau)+\delta_{y}\right)$. The simplest method to find $\boldsymbol{\gamma}^{s p}$, is to initialize (15) with $\left(\alpha_{x}^{s p}, \alpha_{y}^{s p}\right)$ and integrate through $\Delta t$, then initialize (15) with $\left(\beta_{x}^{s p}, \beta_{y}^{s p}\right)$ and integrate through $\Delta t$. We then project back to $\gamma_{x}^{s p}$ by letting $\alpha_{x}^{s p}=\beta_{x}^{s p}=\gamma_{x}^{s p}$. We continue this procedure, integrating each pair of (15) and 
projecting back to $\gamma_{x}^{s p}$ until $\beta_{y}^{s p}-\alpha_{y}^{s p}<\varepsilon$, where $\varepsilon$ is the desired accuracy. We found that for the size of the basin used in these computations, $\varepsilon=1 \mathrm{~km}$ was sufficient. The stagnation point is then given by $\boldsymbol{\gamma}^{s p}=\left(\gamma_{x}^{s p}, \frac{1}{2}\left(\alpha_{y}^{s p}+\beta_{y}^{s p}\right)\right)$. This entire procedure can be repeated at each time step to track the vertical position of the instantaneous stagnation point as a function of time along the boundary. To accelerate convergence, a bisection method can be employed. Once the stagnation point is straddled, we initialize (15) with our estimate for $\gamma^{s p}(\tau)$ and integrating through $\Delta t$. If this trajectory moves toward $\alpha_{y}^{s p}$, that is, $\left|\gamma_{y}^{s p}(\tau)-\alpha_{y}^{s p}\right|<\delta_{y}$, then the actual location of $\gamma_{y}^{s p}(\tau)$ must be between $\alpha_{y}^{s p}$ and $\gamma_{y}^{s p}(\tau)$. Thus, we let $\beta_{y}^{s p}=\gamma_{y}^{s p}(\tau)$ and $\gamma_{y}^{s p}(\tau)=\left(\alpha_{y}^{s p}+\beta_{y}^{s p}\right) / 2$. If $\left|\gamma_{y}^{s p}(\tau)-\alpha_{y}^{s p}\right|>\delta_{y}$, then the actual location of $\gamma(\tau)$ must be between $\beta_{y}^{s p}$ and $\gamma_{y}^{s p}(\tau)$. In that case, we would let $\alpha_{y}^{s p}=\gamma_{y}^{s p}(\tau)$ and again $\gamma_{y}^{s p}(\tau)=\left(\alpha_{y}^{s p}+\beta_{y}^{s p}\right) / 2$. We then initialize (15) with the new $\boldsymbol{\gamma}^{s p}(\tau)$ and repeat the entire procedure until satisfactory convergence is obtained, i.e. $\beta_{y}^{s p}-\alpha_{y}^{s p}<\varepsilon$.

Once we know the basic behavior and range of travel of the stagnation point, we can find and track the hyperbolic trajectory. The procedure for doing this is very similar to the first procedure described for finding the stagnation point. We know that the movement of the hyperbolic trajectory will always be less than or interior to the movement of the stagnation point, so we may let $\left(\alpha_{x}, \alpha_{y}\right)=\left(\gamma_{x}^{s p},\left.\gamma_{y}^{s p}\right|_{\text {min }}\right)$ and $\left(\beta_{x}, \beta_{y}\right)=\left(\gamma_{x}^{s p},\left.\gamma_{y}^{s p}\right|_{\text {max }}\right)$, where $\left.\gamma_{y}^{s p}\right|_{\text {min }}$ and $\left.\gamma_{y}^{s p}\right|_{\text {max }}$ are the minimum and maximum values of $\gamma_{y}^{s p}$ over the time span that we wish to track the hyperbolic trajectory. We are thus certain that the hyperbolic trajectory is straddled and integrate from $\tau$ to $\tau+\Delta t$, then project back to the boundary by letting $\alpha_{x}=\beta_{x}=\gamma_{x}^{s p}$. Note that in the previouslydescribed procedure for the stagnation point we were integrating through $\Delta t$, but the streamfunction which was used for the right-hand side of (15) was frozen at $t=\tau$, so we were finding the instantaneous location of the stagnation point. To find the hyperbolic trajectory, we continue integrating forward in time, i.e. from $\tau+\Delta t$ to $\tau+2 \Delta t$, again letting $\alpha_{x}=\beta_{x}=\gamma_{x}^{s p}$ afterward. This is an important, albeit subtle, difference in the procedures for tracking stagnation points $v s$. hyperbolic trajectories. We continue this method, always integrating forward in time and projecting back to $\gamma_{x}^{s p}$ until $\beta_{y}-\alpha_{y}<\varepsilon$, at which point we have found the hyperbolic trajectory. Depending on the character of the flow along the boundary, $\alpha_{y}$ and $\beta_{y}$ may converge very rapidly, or may take a considerable amount of time. Once $\alpha_{y}$ and $\beta_{y}$ have converged to a satisfactory accuracy, though, we then let $\gamma=\left(\gamma_{x}^{s p}, \frac{1}{2}\left(\alpha_{y}+\beta_{y}\right)\right)$ and just integrate $\boldsymbol{\gamma}$ directly, and of course, project back to the boundary after each integration step. Note that it is not possible to accelerate the procedure for finding and tracking the hyperbolic trajectory with a bisection or similar type of method.

Once the hyperbolic trajectories can be tracked on each boundary, we may use the techniques discussed in $\S 4.2$ to construct invariant manifolds corresponding to these hyperbolic trajectories. Numerically speaking, a stable or unsta- ble invariant manifold is an ordered array of points, i.e. $\boldsymbol{w}_{1}^{s}$, $\boldsymbol{w}_{2}^{s}, \ldots, \boldsymbol{w}_{N}^{s} \in \mathbf{W}^{s}$ and $\boldsymbol{w}_{1}^{u}, \boldsymbol{w}_{2}^{u}, \ldots, \boldsymbol{w}_{M}^{u} \in \mathbf{W}^{u}$, which follows directly from Rule 1 in $\S 3.3$. $N$ and $M$ are the number of points in the stable and unstable manifolds, respectively. The location of these points are computed by first finding the location of the hyperbolic trajectory $\gamma$ at the present time and then setting $\boldsymbol{w}_{1}^{s ; u}=\boldsymbol{\gamma}$. We then integrate $\boldsymbol{w}_{1}^{s ; u}=\boldsymbol{\gamma}$ through $\Delta t^{n p}$, where $\Delta t^{n p}$ is the temporal resolution of the manifold, and then let $\boldsymbol{w}_{2}^{s ; u}=\boldsymbol{\gamma}$, integrate both $\boldsymbol{w}_{1}^{s ; u}=\boldsymbol{\gamma}$ and $\boldsymbol{w}_{2}^{s ; u}=\gamma$ through $\Delta t^{n p}$, so that $N$ increases by 1 each $\Delta t^{n p}$ and thus the manifold continues to grow in length. Empirically, we found that for all values of $\tau_{0}$ that we studied, $\Delta t^{n p}=24$ hours was sufficient for the western boundary, and $\Delta t^{n p}=168$ hours was sufficient for the eastern boundary. Inserting points at regular intervals ensures that the temporal resolution is sufficient, but the manifold will experience rapid stretching as it grows in length, so it is necessary to also check the distance between adjacent points in the manifold and to insert new points between adjacent points if necessary to ensure that sufficient spatial resolution is maintained. There are many methods for checking the distance between points and inserting new points. Each has its advantages depending on the accuracy required of the manifold and the level of algorithmic complexity that one wishes to tackle. The simplest approach is to consider the manifold as a polyline, i.e. a collection of straight line segments and simply check the linear distance between adjacent points and if necessary, insert a new point midway along this segment. The advantage of using this linear approach is that it is straightforward to implement. The disadvantage is that a high spatial resolution is necessary, thus requiring a large value for $N$. Another option is to use circular arcs, fitted through each consecutive triplet of points along the manifold. The angle subtended by the circular arc can then be used to determine if a new point should inserted between existing adjacent points along the manifold. The circular arc method is a bit more difficult to implement, but it has the advantage of generally requiring less points to be maintained in the manifold. The reason for this is that more points are needed along the manifold where it has high curvature and less points are needed where the manifold has lower curvature. Using the subtended angle of the circular as a criteria for point insertion automatically accomplishes this objective. A more accurate method yet, is to describe the manifold as a series of cubic splines, fit through each of four adjacent points in the manifold. The curvature along the spline can then be computed as a function of the arc length along the spline (Spivak, 1979, p. 11), and used to determine whether or not a new point should be inserted between the two existing middle points of the four. We experimented with all three of these methods, but eventually decided to use the linear approach for all of the manifold computations in this paper. Note that whenever it is necessary to insert a new point between existing points, say between $\boldsymbol{w}_{j}^{s ; u}$ and $\boldsymbol{w}_{j+1}^{s ; u}$, it is impractical to shift all of the points in the array $\boldsymbol{w}_{j+1}^{s ; u}, \boldsymbol{w}_{j+2}^{s ; u}, \ldots, \boldsymbol{w}_{N}^{s ; u}$ up one index to make room for the new point. Instead, a "link list" can be 
maintained which describes the physical order of the points in the array $\boldsymbol{w}_{j}^{s ; u}$, which is a functional extension of Rule 1. An entry in the link list, say $\ell_{j}$ is equal to the index value of the point which physically follows the point $\boldsymbol{w}_{j}^{s ; u}$ in the manifold. If the manifold was generated strictly by inserting points at the hyperbolic trajectory, then the link list would be trivial, $\ell_{j}=\ell_{j+1}$. In fact, when a point is inserted at the hyperbolic trajectory, we simply set $\boldsymbol{w}_{N+1}^{s ; u}=\boldsymbol{\gamma}$ and $\ell_{N}=N+1$, and then increment $N$, which generates this trivial link list. To insert a new point between say $\boldsymbol{w}_{j}^{s ; u}$ and $\boldsymbol{w}_{\ell_{j}}^{s ; u}$, we set $\boldsymbol{w}_{N+1}^{s ; u}=\frac{1}{2}\left(\boldsymbol{w}_{j}^{s ; u}+\boldsymbol{w}_{\ell_{j}}^{s ; u}\right)$, and then set $\ell_{N+1}=\ell_{j}$ and $\ell_{j}=N+1$, in that order.

\subsection{Pips and lobes}

Once we have computed both stable and unstable manifolds, it is next necessary to determine the pips of the manifolds and the resultant lobes. If each manifold is considered a polyline described by the arrays $\boldsymbol{w}_{1}^{s}, \boldsymbol{w}_{2}^{s}, \ldots, \boldsymbol{w}_{N}^{s}$ for the stable manifold and $\boldsymbol{w}_{1}^{u}, \boldsymbol{w}_{2}^{u}, \ldots, \boldsymbol{w}_{M}^{u}$ for the unstable manifold, then all of the intersection points can be determined by computing

$p_{x}=\left(m^{u} w_{x, j}^{u}-m^{s} w_{x, i}^{s}+w_{y, i}^{s}-w_{y, j}^{u}\right) /\left(m^{u}-m^{s}\right)$,

$p_{y}=m^{u}\left(p_{x}-w_{x, j}^{u}\right)+w_{y, j}^{u}$,

where $i=1,2, \ldots, N$ and $j=1,2, \ldots, M, m^{s ; u}=$ $\left(w_{y, \ell_{i}^{s ; u}}^{s ; u}-w_{y, i}^{s ; u}\right) /\left(w_{x, \ell_{i}^{s ; u}}^{s ; u}-w_{x, i}^{s ; u}\right)$. The point $\boldsymbol{p}$ is the unique intersection point of the line defined by $\boldsymbol{w}_{i}^{s}$ and $\boldsymbol{w}_{\ell_{i}^{s}}^{s}$, and the line defined by $\boldsymbol{w}_{j}^{u}$ and $\boldsymbol{w}_{\ell_{j}^{u}}^{u}$, which is an intersection only if

$$
\begin{aligned}
& \left|\boldsymbol{p}-\boldsymbol{w}_{i}^{s}\right| \leq\left|\boldsymbol{w}_{\ell_{i}^{s}}^{s}-\boldsymbol{w}_{i}^{s}\right|, \quad\left|\boldsymbol{p}-\boldsymbol{w}_{\ell_{i}^{s}}^{s}\right| \leq\left|\boldsymbol{w}_{\ell_{i}^{s}}^{s}-\boldsymbol{w}_{i}^{s}\right| \\
& \left|\boldsymbol{p}-\boldsymbol{w}_{j}^{u}\right| \leq\left|\boldsymbol{w}_{\ell_{j}^{u}}^{u}-\boldsymbol{w}_{j}^{u}\right|, \quad\left|\boldsymbol{p}-\boldsymbol{w}_{\ell_{j}^{u}}^{u}\right| \leq\left|\boldsymbol{w}_{\ell_{j}^{u}}^{u}-\boldsymbol{w}_{j}^{u}\right| .
\end{aligned}
$$

Note that finding all of the intersection points is an $N \times M$ procedure, so as the manifolds grow in length, it can become computationally expensive. There is a method for reducing the computational expense of finding intersection points far below $N \times M$ operations, but describing it is beyond the scope of this paper. It is described by Coulliette et al. (2000). Once all of the intersection points are found, they must be classified as either primary (pips) or secondary (sips). An intersection point can be determined to be a pip by applying (16) and (17), and checking that the segment of the unstable manifold from the hyperbolic trajectory of the western boundary does not intersect the segment of the stable manifold from the intersection point to the hyperbolic trajectory on the eastern boundary. After the intersection points are classified, a lobe is defined simply by the segment of the unstable manifold and the segment of the stable manifold between each adjacent pair of pips. Numerically speaking, the lobes themselves are simply a concatenation of two series of points, each from a link list array. The set of points describing the perimeter of each lobe can then be used for visualization, i.e. colorizing the lobe, or computing the areas of the lobes, and thus transport quantities, using Green's Theorem. Using Green's Theorem is also useful for determining if a given lobe has or will move across the boundary from south to north, or if it has or will move across the boundary from north to south. If we always apply Green's Theorem by integrating along the stable manifold in the direction of incrementing index value, then the integral will either be positive or negative. Lobes with negative area integrals have or will move across the boundary from south to north, and vice versa. Lobes with positive area integrals have or will move across the boundary from north to south.

Identifying lobes should not be thought of as a static procedure, i.e. as an analysis only of the present manifolds. Rather it needs to be considered a dynamical procedure. In order to completely harness the theoretical potentials of lobe dynamics for flows of general time dependence, it is necessary to be able to find all of the lobes that result from a stable and unstable manifold, and then at a later time to find again the lobes that result from the same stable and unstable manifold. The process is continued a short time later, again finding the lobes that results from another realization of the manifolds, etc. Once the lobes have been identified at each realization of the manifolds, they must be matched from realization to realization, so that it can be determined which lobe at a given time is actually the same lobe at a later time. This follows directly from Rule 2 of $\S 3.3$. Developing a robust algorithm for lobe matching is an essential, yet challenging task, especially when numerical errors in the computed manifolds and hence, intersection points must be considered. Describing such an algorithm is beyond the scope of this paper, but is discussed in detail by Coulliette et al. (2000).

\section{Results}

In the following section we present results on intergyre transport in the top layer for different values of the wind curl stress. In each simulation the fluid starts from rest and is allowed to spin up for 25,000 days.

\section{$5.1 \tau_{0}=0.140:$ A time-periodic velocity field}

For this value of the wind stress a time-periodic velocity field is obtained. Evidence for this is presented in Fig. 8. In the top panel of Fig. 8 the spatially-averaged kinetic energy as a function of time is shown for the three layers, which are denoted by the curves $K E_{1}, K E_{2}$, and $K E_{3}$, respectively, for $\tau_{0}=0.140 \mathrm{dyn} / \mathrm{cm}^{2} . K E_{T}$ denotes the total kinetic energy. We see from the top panel of Fig. 8 that $K E_{T}$ is nearly identical to $K E_{1}$, so for this value of $\tau_{0}$, and also for the other values of $\tau_{0}$ studied in this paper, most of the energy remains in the top layer of the model. In the lower panel of Fig. 8 we show the power spectrum of the time varying part of the spatially-averaged kinetic energy in the first 

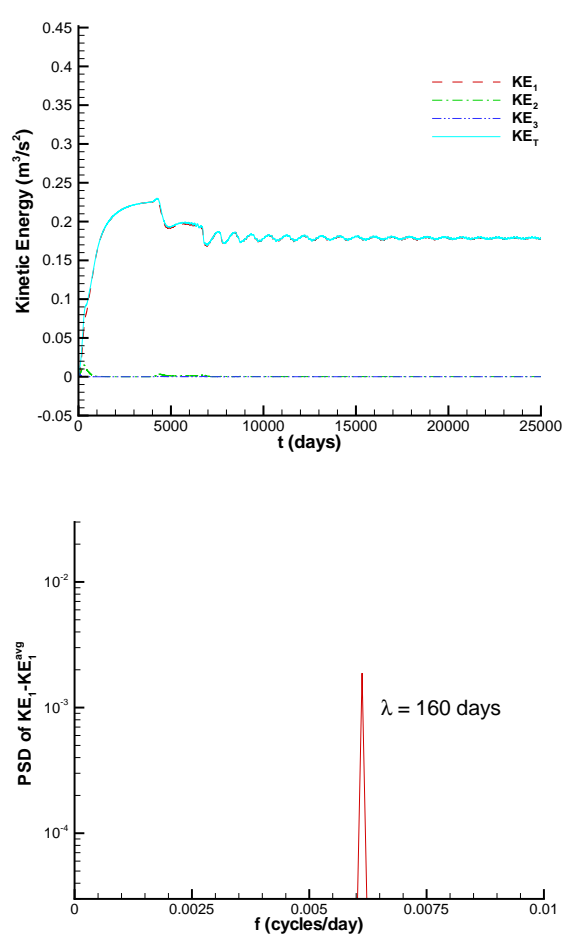

Fig. 8. Spatially-averaged kinetic energy and the corresponding power spectrum for the upper layer when $\tau_{0}=0.140 \mathrm{dyn} / \mathrm{cm}^{2}$, indicating a period of 160 days.

layer after the 25,000 day spin up, $K E_{1}-K E_{1}^{a v g}$, where $K E_{1}^{a v g}$ denotes the time average of $K E_{1}$. The average is computed from day 25001 until day 29000 (4000 days total). From the spectrum, we see that the top layer has a period of $\tilde{T}=160$ days. The spin up time of 25000 days is much longer than typically used in other work on quasigeostrophic models. The objective in using such a long spin up was to be certain that we had reached a statistically steady state. We found that the spin up time necessary to reach a statistically steady state varies inversely with the magnitude of $\tau_{0}$. In this study we prescribe $0.140 \mathrm{dyn} / \mathrm{cm}^{2} \leq \tau_{0} \leq 0.170 \mathrm{dyn} / \mathrm{cm}^{2}$, which is low in magnitude relative to a more typical value of $\tau_{0}=1.0 \mathrm{dyn} / \mathrm{cm}^{2}$ used in the other literature on quasigeostrophic models (e.g. Cummins and Mysak, 1988; Lozier and Riser, 1989, 1990).

Before we can compute the manifolds and lobes, we must first be able to track the hyperbolic trajectories along the western and eastern boundaries. Fig. 9 demonstrates the result of the numerical method described in $\S 4.3$ for tracking hyperbolic trajectories on boundaries. The upper panel shows the vertical position of the hyperbolic trajectory on the western boundary as a function of time and the lower panel shows the vertical position of the hyperbolic trajectory on the eastern boundary as a function of time. In both panels, the blue line is $\alpha_{y}$, the lower bound on the hyperbolic trajectory, and the green line is $\beta_{y}$, the upper bound on the hyperbolic
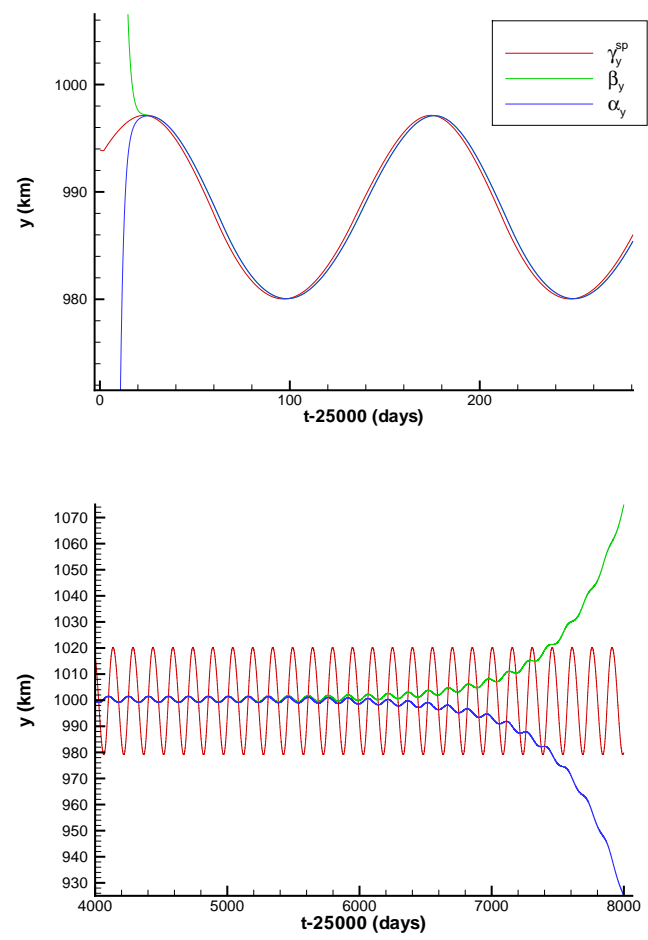

Fig. 9. The location of the hyperbolic trajectory and the instantaneous location of the stagnation point for the western boundary (upper panel) and the eastern boundary (lower panel) when $\tau_{0}=0.140 \mathrm{dyn} / \mathrm{cm}^{2}$.

trajectory. Also included in both panels is a red line, which is the instantaneous vertical location of the stagnation point, $\gamma_{y}^{s p}$. Note that in the upper panel, $\alpha_{y}$ and $\beta_{y}$ converge rapidly to each other, that is $\beta_{y}-\alpha_{y}<1 \mathrm{~km}$ at $t=25021$ days, and then follow a path very similar, yet distinct, from that of $\gamma_{y}^{s p}$. As mentioned in $\S 4.3$, we see from Fig. 9 that once $\alpha_{y}$ and $\beta_{y}$ have converged, the range of travel of the hyperbolic trajectory is bounded by the range of travel of the instantaneous location of the stagnation point, i.e. $\left.\gamma_{y}^{s p}\right|_{\min } \leq \alpha_{y} \leq\left.\gamma_{y}^{s p}\right|_{\max }$ and $\left.\gamma_{y}^{s p}\right|_{\min } \leq \beta_{y} \leq\left.\gamma_{y}^{s p}\right|_{\max }$. This principal also applies to the lower panel of Fig. 9, but there are some distinctions between the upper and lower panel. The hyperbolic trajectory does not follow a path that is nearly identical to the stagnation point, as in the upper panel. In fact, its range of travel is considerably less than that of the stagnation point. Also, $\alpha_{y}$ and $\beta_{y}$ converge much more slowly in the lower panel, requiring over 2000 days to converge to $1 \mathrm{~km}$ or less. This is a result of the velocities near the eastern boundary being much slower than those near the western boundary. In both panels, though, we see that the hyperbolic trajectory changes direction, that is, the sign of the vertical velocity of the hyperbolic trajectory changes, as the instantaneous location of the stagnation point crosses to the opposite side of the hyperbolic trajectory.

Once we are able to track the hyperbolic trajectories on 

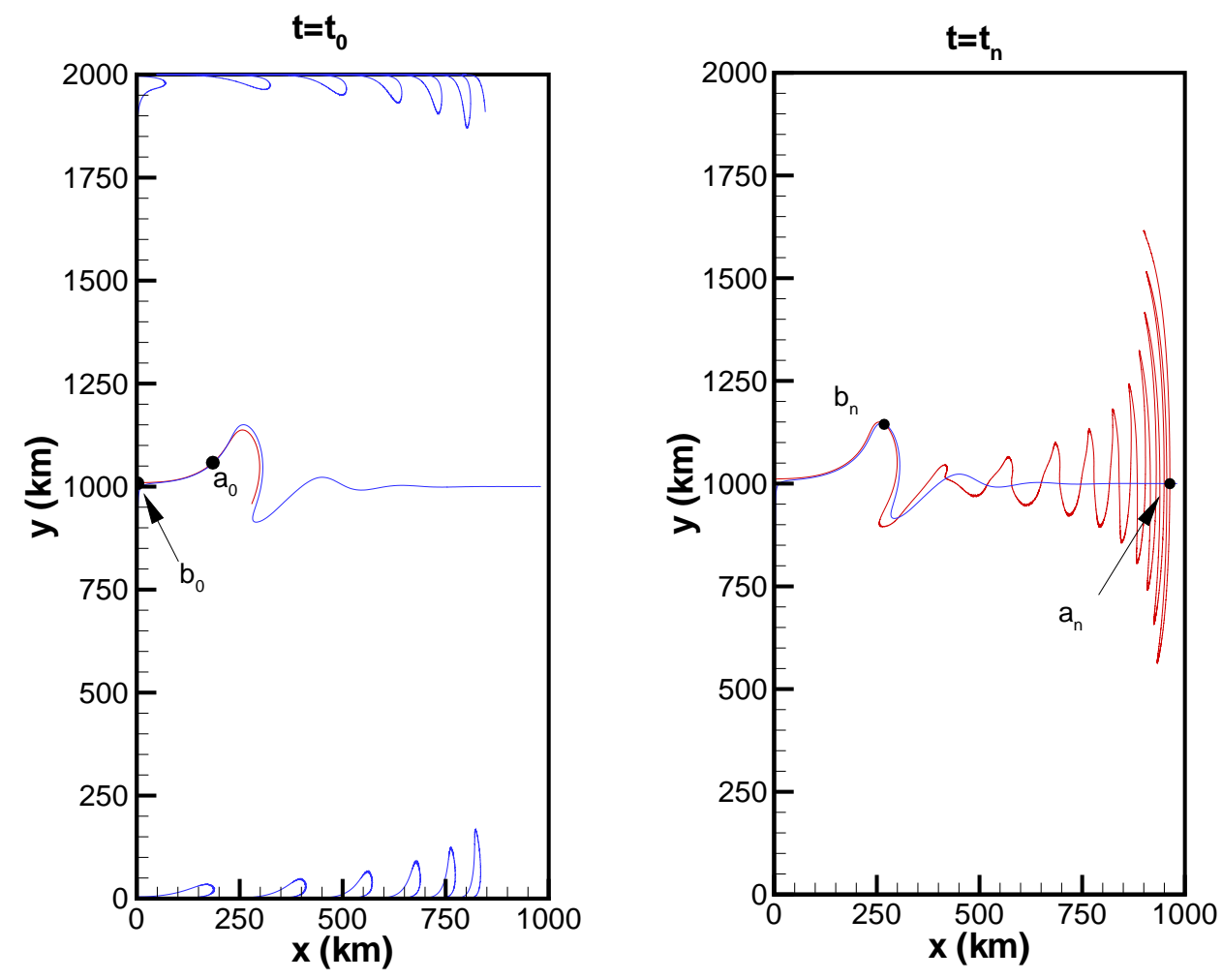

Fig. 10. Lobes created by the intersection of the stable and unstable manifolds of the hyperbolic trajectories on the eastern and western boundaries, respectively, for $\tau_{0}=0.140 \mathrm{dyn} / \mathrm{cm}^{2}$.

both boundaries, we can compute the associated manifolds. In Fig. 10 we show the lobes created by the intersecting stable and unstable manifolds of the hyperbolic trajectories on the eastern and western boundaries, respectively, for $\tau_{0}=0.140 \mathrm{dyn} / \mathrm{cm}^{2}$. These are the manifolds that govern intergyre transport. In the left panel of Fig. 10, we show the manifolds at time $t_{0}=25075$ days, that is, 75 days after the spin up. Two points are labeled in the panel, $a_{0}$ and $b_{0}$, which are pips of the unstable and stable manifolds. In addition, $a_{0}$ is the bip for time $t=t_{0}$, which means that the boundary between the northern and southern gyre is defined by the red unstable manifold to the left of $a_{0}$ and the blue stable manifold to the right of $a_{0}$. Between $a_{0}$ and $b_{0}$ is a series of eighteen others pips and corresponding lobes, nine of which are in the northern gyre and ten of which are in the southern gyre. The right panel of Fig. 10 shows what happens to the manifolds and lobes ten periods later, i.e. when $t=t_{n}=26635$ days. Note that $a_{0}$ has mapped to $a_{n}$ and $b_{0}$ has mapped to $b_{n}$. Now, $b_{n}$ is the new bip and the lobes between $a_{n}$ and $b_{n}$ illustrate the transport that has occurred between the northern and southern gyre during the time interval $t_{n}-t_{0}$. So, in the left panel, the lobes which are shown there indicate the fluid which will be transported across the boundary between the northern and southern gyre, while in the right panel, the lobes indicate the fluid which has been transported across the boundary.

Not only do the lobes illustrate the location and shape of the fluid which will be or has been transported across the boundary, but, in addition, they can be used to precisely calculate the amount of transported fluid. For example, we use Green's Theorem to compute the areas of each lobe, sum the areas of the lobes between $a_{n}$ and $b_{n}$ on the north side of the boundary, multiply by the upper layer thickness, $H_{1}$, and then divide by $t_{n}-t_{0}$, which gives $\phi_{S, N}=0.0590 \mathrm{~Sv}$. This represents the amount of irreversible flux from the southern gyre into the northern gyre during the interval between $t_{0}$ to $t_{n}$. Likewise, we perform a similar calculation for the lobes in the southern gyre to get $\phi_{N, S}=0.0563 \mathrm{~Sv}$. If we subtract this value from $\phi_{S, N}$ we get $\phi_{R}=2.67 \times 10^{-3} \mathrm{~Sv}$, which denotes the instantaneous value of the reversible component of the flux at $t=t_{n}$ described in the previous section, which is attributed to the movement of the boundary between the southern and northern gyres from $t_{0}$ to $t_{n}$. For a point of reference regarding these transport quantities, we can compute the transport along the jet, $\phi_{J}$, by taking the difference in the instantaneous streamfunction values at $t=t_{n}$ from the centers of the eddies above and below the jet near the western boundary and multiplying by the distance between the two centers.

In this case, $\phi_{J}=6.12 \mathrm{~Sv}$ is two orders of magnitude larger than $\phi_{S, N}$ and five orders of magnitude larger than $\phi_{R}$. However, it is important to explore and estimate the numerical accuracy of the flux computations which rely on the lobes. To do this, we can exploit certain behaviors of 

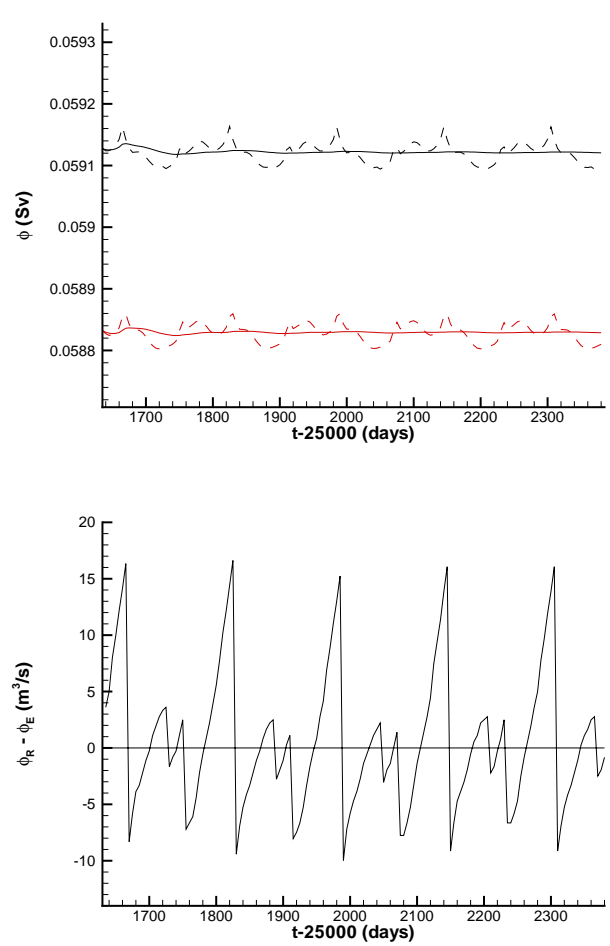

Fig. 11. Flux as a function of time where $\tau_{0}=0.140 \mathrm{dyn} / \mathrm{cm}^{2}$. The flux $\phi_{S, N}$ is computed from lobes which transported fluid from the southern gyre into the northern (black dashed line) and the flux $\phi_{N, S}$ is computed from the lobes which transported fluid from the northern gyre into the southern (red dashed line). Their time averages $\bar{\phi}_{S, N}$ and $\bar{\phi}_{N, S}$ are shown by the solid black and red lines, respectively.

this particular case. First, in many time-periodic solenoidal fields, such as this case, all of the lobes on a given side of the boundary should theoretically be identical in area. So, we compute the variation, $\phi_{v}$, in the lobe area for all the lobes on the north side of the boundary by subtracting the minimum and maximum lobe areas, dividing the result by the maximum lobe area, and multiplying by $100 \%$. In this case, $\phi_{v}=0.429 \%$, which serves as a measure of the global relative error. Another measurement of the global relative error takes a bit more effort to obtain, but the necessary computations yields other insights into the physics of the transport processes.

If we re-compute the manifolds and corresponding flux computations for Fig. 10, but start and stop the computations five days later, that is, $t_{0}=25085$ days and $t_{n}=$ 26635 days, and maintain the same number of points in each manifold, 18000 in the unstable, and 3000 in the stable, then we will get slightly different values for $\phi_{S, N}, \phi_{N, S}$ and $\phi_{R}$. If we repeat this computation at five day intervals for 750 days, the result is Fig. 11. Note that $\phi_{S, N}$ is the black dashed line and $\phi_{N, S}$ is the red dashed line in the upper panel of Fig. 11. The red and black solid lines in the upper panel are $\bar{\phi}_{S, N}$ and
$\bar{\phi}_{N, S}$, which represent the time average of $\phi_{S, N}$ and $\phi_{N, S}$ from the present $t$ back to $t_{0}$, respectively. Since $\phi_{S, N}-\phi_{N, S}$ represents the reversible flux due boundary movement, and flow is time-periodic, then $\bar{\phi}_{S, N}$ and $\bar{\phi}_{N, S}$ should approach the same constant value when $t_{n}-t_{0}>>\tilde{T}$. In this case, $t_{0}=25080$ days, $t_{n}=27385$ days, and $\tilde{T}=160$ days, which satisfies this condition. From Fig. 11 we can see that although $\bar{\phi}_{S, N}$ and $\bar{\phi}_{N, S}$ both approach constant values over the 750 day span shown, there is a small difference between their final values, i.e. $\phi_{E}=\left|\bar{\phi}_{S, N}-\bar{\phi}_{N, S}\right|_{t=27385}$. This difference is another measure of the global error of the computations, which in this case is $0.387 \%$. So, by the value given for $\phi_{v}$ and this difference between $\bar{\phi}_{S, N}$ and $\bar{\phi}_{N, S}$, it is safe to say that for the parameters chosen to control the accuracy of the numerical methods, such as the temporal interpolation, spatial interpolation, integration, spatial resolution of the manifolds, etc., chosen for the computations in these studies, the overall global error is less than $1 \%$. The lower panel of Fig. 11 shows $\phi_{R}$, corrected by $\phi_{E}$. Note that $\phi_{R}$ is truly reversible, in the sense that it oscillates above and below the zero line, and that it has a period of 160 days, equal to the period of the spatially-averaged kinetic energy of the entire basin shown in the lower panel of Fig. 8. Note that $\phi_{R}$ is a very small quantity relative to that of $\phi_{S, N}$ or $\phi_{N, S}$, so the boundary between the northern and southern gyres is nearly stationary and nearly all of the flux across the boundary is of a long-term irreversible nature. The discontinuities in the curvature of $\phi_{S, N}, \phi_{N, S}$, and $\phi_{R}$ are a natural consequence of the discrete changes in the bip at $t=t_{n}$ as the manifolds are advanced forward in time.

\section{$5.2 \tau_{0}=0.160:$ A time-periodic velocity field}

Since our objective is to develop methods and to study flows with periodic, quasiperiodic and chaotic time dependence, it is important to increment $\tau_{0}$ gradually and examine the resulting implications on the lobe dynamics. If we increase the magnitude of the wind stress to $\tau_{0}=0.160 \mathrm{dyn} / \mathrm{cm}^{2}$, the flow remains time-periodic. Evidence for this is presented in Fig. 12. The spectrum of the mean spatial kinetic energy of the top layer in the basin is similar to that shown in Fig. 8, but indicating a period of 154 days.

In Fig. 13 we show the lobes created by the intersecting stable and unstable manifolds of the hyperbolic trajectories on the eastern and western boundaries, respectively, for $\tau_{0}=0.160 \mathrm{dyn} / \mathrm{cm}^{2}$ at $t=t_{n}=26580$ days. There are 26 intersections of the unstable and stable manifold segments shown, all of which are pips. As in the right panel of Fig. 10, two of the pips are labeled, $a_{n}$ and $b_{n} . b_{n}$ is the present bip and $a_{n}$ is the map of $a_{0}$ from $t=t_{0}=25075$ days. The two manifolds and 26 pips define 13 lobes in the northern gyre, and 12 lobes in the southern gyre. The overall geometric pattern of the lobes is very similar to the previous $\tau_{0}=0.140$ case. In fact, for all values of $\tau_{0}$ the motion of the lobes follows the same general pattern. Note the lobes at the top of the figure. Under time evolution they move to the left, toward the western boundary. After reaching the western 

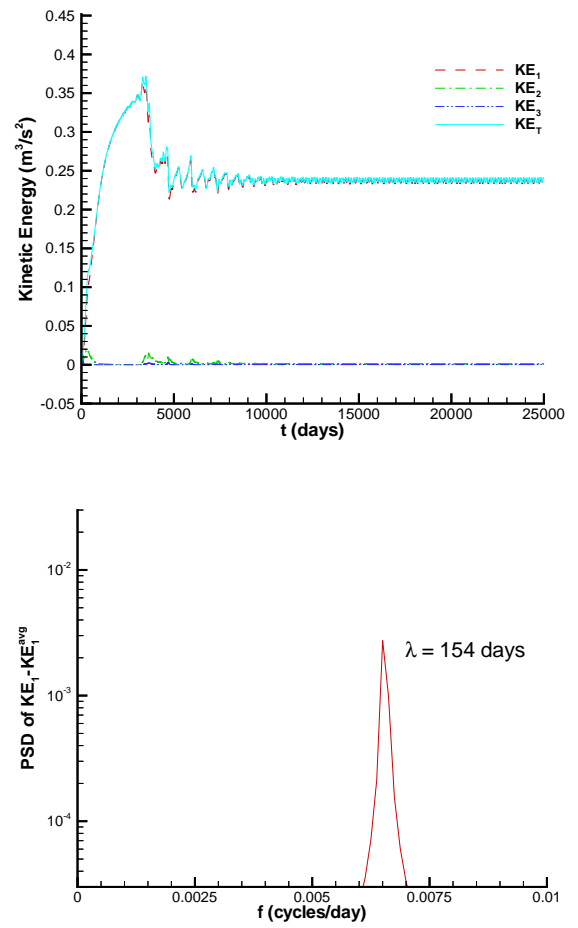

Fig. 12. Spatially-averaged kinetic energy and the corresponding power spectrum for the upper layer when $\tau_{0}=0.160 \mathrm{dyn} / \mathrm{cm}^{2}$, indicating a period of 154 days.

boundary they move downwards along the western boundary until they reach a neighborhood of the hyperbolic trajectory on the western boundary. Then they move back to the interior of the flow, and in the process, cross from the northern to the southern gyre. However, in this case of $\tau_{0}=0.160$, the lobes move around the gyre more rapidly than in the $\tau_{0}=0.140$ case.

For the lobes shown in Fig. 13, the intergyre transport is $\phi_{S, N}=0.136 \mathrm{~Sv}$ while the transport along the jet is $\phi_{J}=6.98 \mathrm{~Sv}$. So, we see that the intergyre transport has increased significantly (almost by a factor of two). Yet the increase in transport along the jet does not experience as significant an increase. Note also that the asymmetry between the patterns of lobes in the northern and southern gyres is more pronounced than for $\tau_{0}=0.140$. This is due to the fact that the meandering of the jet is more pronounced for $\tau_{0}=0.160$. The instantaneous value of the reversible flux is $\phi_{R}=0.00335 \mathrm{~Sv}$ and the lobe variation for the northern lobes is $\phi_{v}=0.435 \%$, both of which are increased from the their corresponding values when $\tau_{0}=0.140$.

\section{$5.3 \tau_{0}=0.165$ : A time-periodic velocity field}

For this value of the wind stress another time-periodic velocity field is obtained, which is clear from the wind up and power spectrum of the spatially-averaged kinetic energy of the first layer presented in Fig. 14. The period is 151 days.

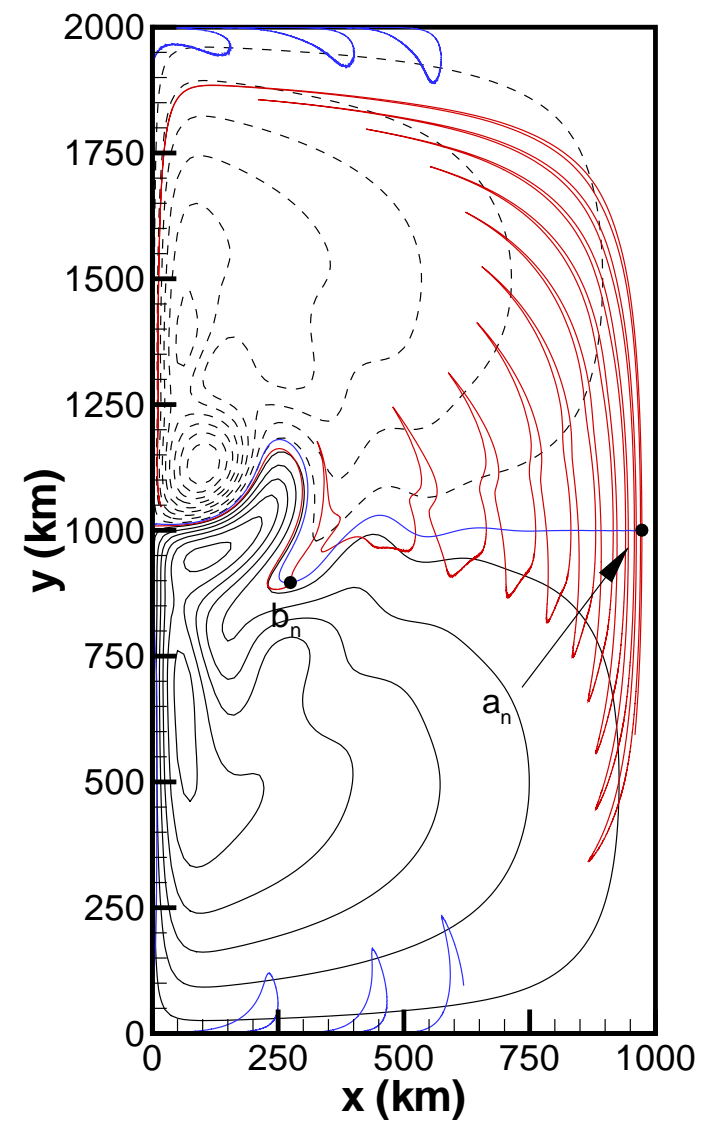

Fig. 13. Lobes created by the intersection of the stable and unstable manifolds of the hyperbolic trajectories on the eastern and western boundaries, respectively, for $\tau_{0}=0.160 \mathrm{dyn} / \mathrm{cm}^{2}$ at $t_{n}=26580$ days.

In Fig. 15, we show the lobes created by the intersecting stable and unstable manifolds of the hyperbolic trajectories on the eastern and western boundaries, respectively, for $\tau_{0}=0.165 \mathrm{dyn} / \mathrm{cm}^{2}$ at $t=27925$ days. The lobes have been colorized with alternating green and purple, so that it is easier to see the lobes. The colorization also makes it easier to see the transport behavior. In Fig. 15, purple lobes can be seen in the southern gyre along the western boundary, in the jet, and in the northern gyre. Green lobes can be seen along in the northern gyre along the western boundary, just past the jet, and in the southern gyre. Since the flow is periodic and one lobe of each color is generated each period, each purple lobe can be thought of as a map of any other purple lobe, and likewise, each green lobe can be thought of as a map of any other green lobe. So, the colorized lobes allow us to "see" the transport process: green lobes descend the western boundary, and then cross the jet into the southern gyre. Purple lobes ascend the western boundary, and then cross the jet into the northern gyre. An animated version of Fig. 15 is available on the authors' web site at http://transport.caltech.edu, along 

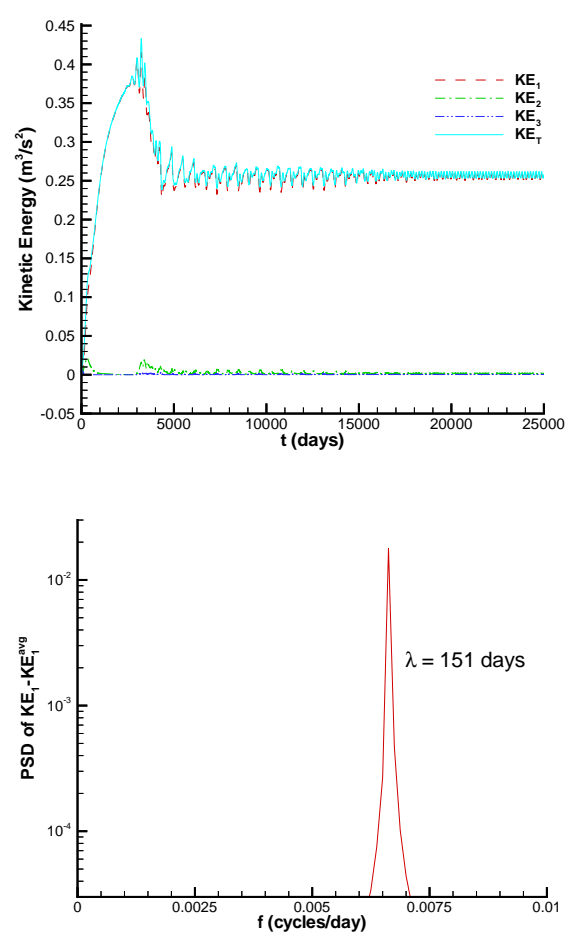

Fig. 14. Spatially-averaged kinetic energy and the corresponding power spectrum for the upper layer when $\tau_{0}=0.165 \mathrm{dyn} / \mathrm{cm}^{2}$, indicating a period of 151 days.

with animations of several of the other similar figures in this paper. The reader is encouraged to view the animations, since they clearly illustrate the intergyre transport process. In addition to elucidating intergyre transport, the lobes also show the geometric shape of fluid both before and after it has crossed the boundary. Note that the geometric pattern of the lobes shows new behavior that is different than that observed for $\tau_{0}=0.140$ and 0.160 .

In particular, one notices that the purple lobes in the northern gyre have developed a ring-like structure due to the rolling up of the tips, which occurs as a result of the interaction with the eddy above the jet.

Fig. 16 shows several steps in the formation of the ring-like structures at a northern meander starting at $t=27945$ days and spanning a 150 day period, at 30 day intervals. Since the flow has a period of 151 days, the last panel in Fig. 16 is nearly identical to the first. In the first panel of Fig. 16, a purple lobe can be seen just after it crossed the jet. The panels which follow show a ring-like structure pinch off from the jet, remaining attached to the remainder of the lobe by a very thin filament. From the last panel, it is evident that only a small amount of the fluid in the ring-like structure is purple, i.e. from the southern gyre. The ring-like structures are similar to the well-known rings often discussed in oceanographic literature, particularly in discussions of transport processes in the vicinity of the Gulf Stream. They are similar in the sense

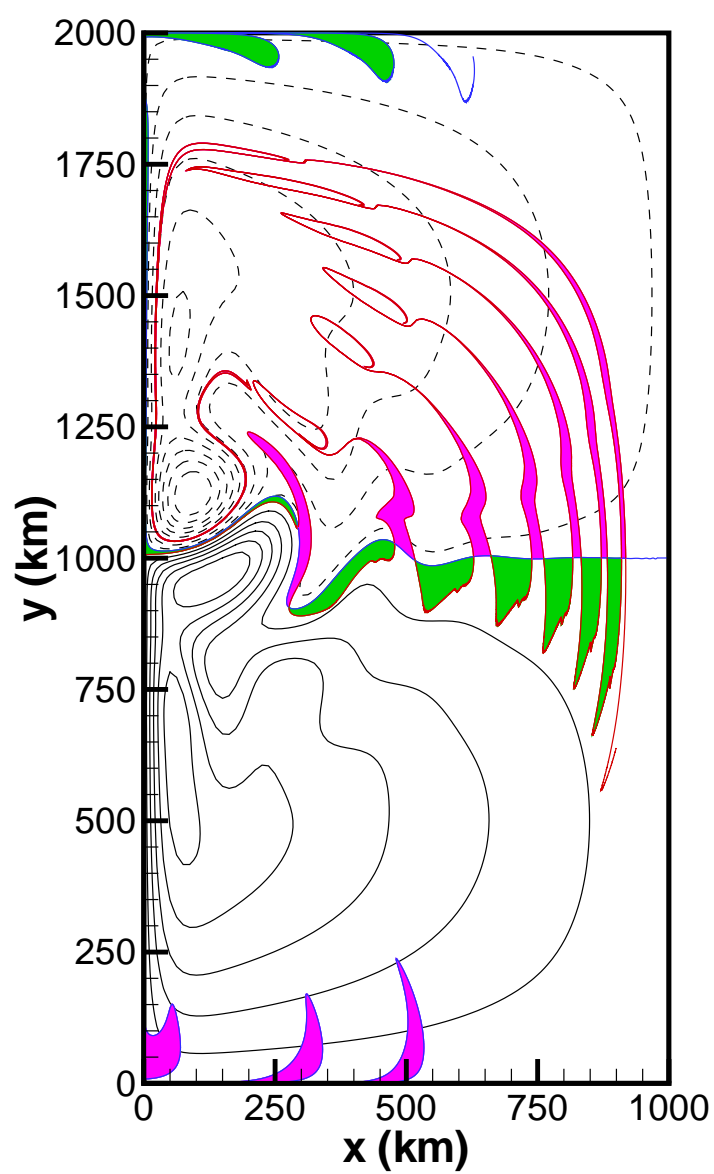

Fig. 15. Lobes created by the intersection of the stable and unstable manifolds of the hyperbolic trajectories on the eastern and western boundaries, respectively, for $\tau_{0}=0.165 \mathrm{dyn} / \mathrm{cm}^{2}$ at $t=27925$ days. To make it easier to see the lobes, they have been colorized with alternating green and purple.

that they are quasigeostrophic coherent Lagrangian structures, but dissimilar in that they do not have associated with them typical velocity and potential vorticity gradients. As a result, their path of travel after they are formed is simply the result of the ambient velocity field, moving slowly around the gyre, as seen in Fig. 15, whereas rings, once they are formed, typically travel in a direction opposite to that of the ambient fluid, e.g. in the case of the Gulf stream. They move in a direction parallel to, but opposite that of the Gulf stream. From some further studies that we have done corresponding to significantly larger values of $\tau_{0}$, the same Lagrangian structures that we see here do develop associated velocity and potential vorticity gradients and have a path of travel parallel and opposite to that of the jet. So, we will refer to these Lagrangian structures as rings throughout the remainder of this paper, since they seem to be the low $\tau_{0}$ analog of classical rings. It is important to realize that because these rings at low $\tau_{0}$ do not have associated velocity or potential vorticity gradients, they are undetectable or invisible by any method other than 

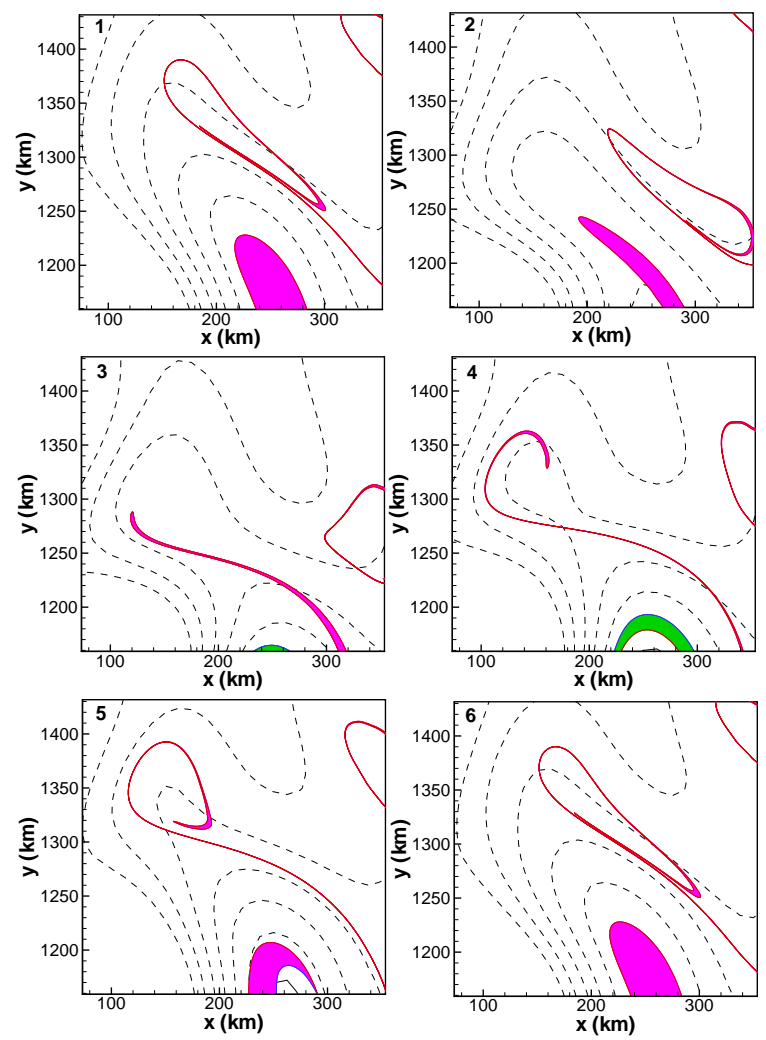

Fig. 16. A series of snapshots showing the formation of a ring in the southern gyre when $\tau_{0}=0.165 \mathrm{dyn} / \mathrm{cm}^{2}$. The upper-left panel starts at $t=27945$ days, and then each following panel depicts the ring formation process at 30 days intervals.

that which we describe using lobe dynamics. In this particular case, note from Fig. 15 and Fig. 16 that the fluid in the interior of the ring is fluid from the northern gyre. Therefore we see that these rings for this value of $\tau_{0}$ do not transport fluid, or at least transport very small amounts of fluid, from the southern to the northern gyre since these lobes originated earlier in the southern gyre. This is an interesting observation since there has been much debate in the literature about the how much the rings contribute to intergyre transport. We will also look at the formation of rings at higher values of $\tau_{0}$ in the following sections to determine if there is a direct relationship between magnitude of the wind stress curl and the amount of intergyre transport contributed by rings.

The intergyre flux computed from the northern lobes shown in Fig. 15 is $\phi_{S, N}=0.152 \mathrm{~Sv}$, the reversible flux is $\phi_{R}=0.00372 \mathrm{~Sv}$, and the flux of the jet is $\phi_{J}=7.18 \mathrm{~Sv}$, all of which represent a linear increase over the values corresponding to $\tau_{0}=0.140$ and $\tau_{0}=0.160$. The variation computed from the northern lobes is $\phi_{v}=0.263 \%$, which is a decrease from the variation corresponding to both $\tau_{0}=0.140$ and $\tau_{0}=0160$. This is probably due to the fact that only seven lobes were computed for this case, whereas ten lobes were computed for both of the previous cases, thus the variation in lobe size is likely to be less.
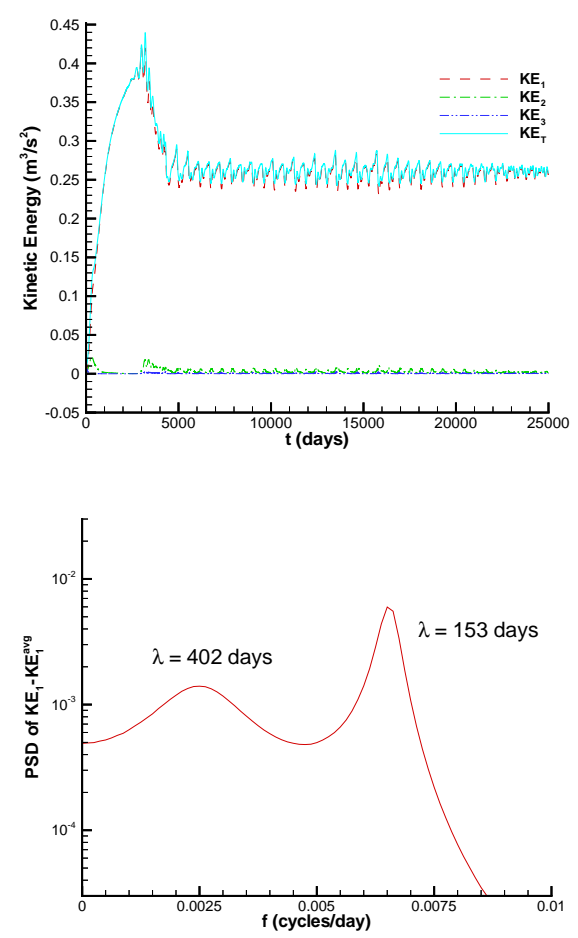

Fig. 17. Spatially-averaged kinetic energy and the corresponding power spectrum for the upper layer when $\tau_{0} \cong 0.1672 \mathrm{dyn} / \mathrm{cm}^{2}$. However, now the spectrum is not dominated by a single period, but rather two periods: one of 153 days and the other 402 days. We refer to this situation as "quasiperiodic".

\section{$5.4 \tau_{0} \cong 0.1672$ : A time-quasiperiodic velocity field}

For this value of the wind stress we obtain a velocity field that is clearly not temporally periodic. We show the spectrum in Fig. 17. We see two dominant peaks in the spectrum: one at 153 days and the other at 402 days, and we refer to this situation as "quasiperiodic". The precise value of the wind stress curl necessary to obtain this quasiperiodic condition is $0.16715087 \mathrm{dyn} / \mathrm{cm}^{2}$, which was not a trivial value to find, requiring the model to be spun up from rest over 60 times.

In Fig. 18 we show the lobes created by the intersecting stable and unstable manifolds of the hyperbolic trajectories on the eastern and western boundaries, respectively, for $\tau_{0} \cong 0.1672 \mathrm{dyn} / \mathrm{cm}^{2}$. There are 18 intersections of the stable and unstable manifold, all of which are pips. The manifolds and pips define 17 lobes, 9 of which are purple and 8 of which are green. Of the purple lobes, 2 of them in the southern gyre along the western boundary indicate fluid which will be transported across the boundary, while 7 of them in the northern gyre indicate fluid which has recently been transported across the boundary. Of the green lobes, 2 of them in the northern gyre along the western boundary indicate fluid which will be transported across the boundary, while 6 of them in the southern gyre indicate fluid which has 


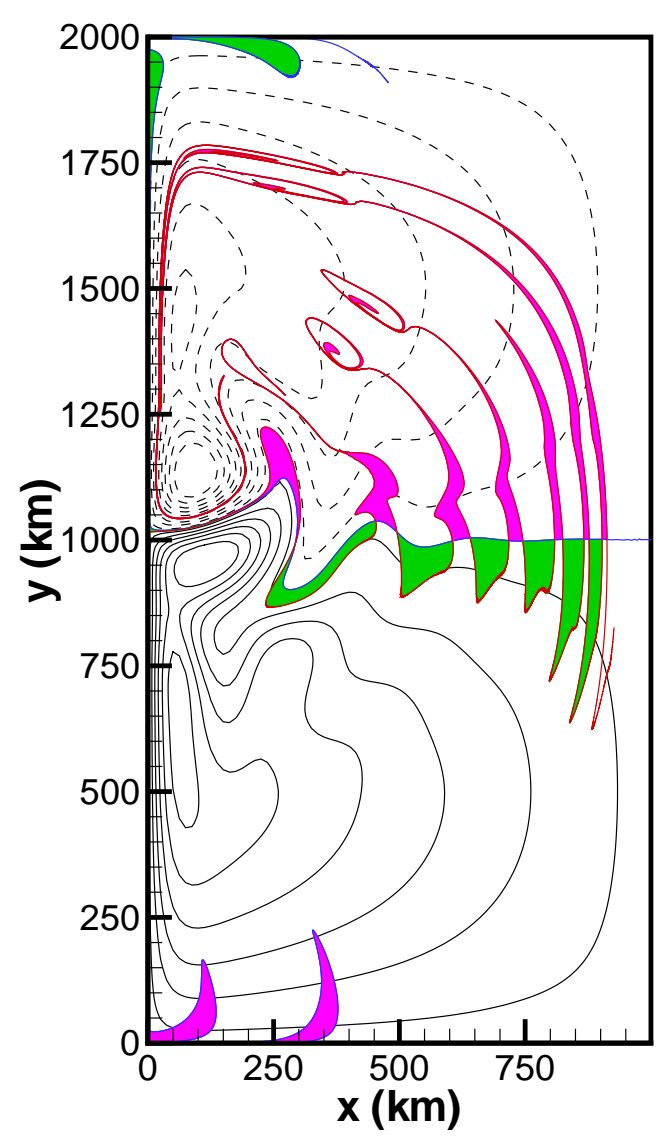

Fig. 18. Lobes created by the intersection of the stable and unstable manifolds of the hyperbolic trajectories on the eastern and western boundaries, respectively, for $\tau_{0} \cong 0.1672 \mathrm{dyn} / \mathrm{cm}^{2}$ at $t=26057$ days. As in Fig. 15, the lobes have been colorized with alternating green and purple.

been recently transported across the boundary. As for the geometry of the lobes, we see some similarity with the situation for $\tau_{0}=0.165$ in that the tips of most of the purple lobes in the northern gyre have rolled up to form rings. However, there is one very important difference. For this case we see a small "ring within the ring". This interior ring is filled with fluid from the southern gyre (but it is surrounded by fluid from the northern gyre). Thus, the lobes rings now contribute to the transport of fluid from the southern to the northern gyre.

The intergyre flux for the lobes shown in Fig. 18 is $\phi_{S, N}=$ $0.160 \mathrm{~Sv}$. The reversible flux is $\phi_{R}=0.0142 \mathrm{~Sv}$ and the flux along the jet is $\phi_{J}=7.28 \mathrm{~Sv}$. Note that both the intergyre flux and the flux along the jet have again increased linearly from the values corresponding to previously discussed magnitudes of $\tau_{0}$. But the reversible flux has increased nonproportionally. We should note that although the reversible flux values have thus far increased with increasing $\tau_{0}$, these are instantaneous values only, and are indicative only of the
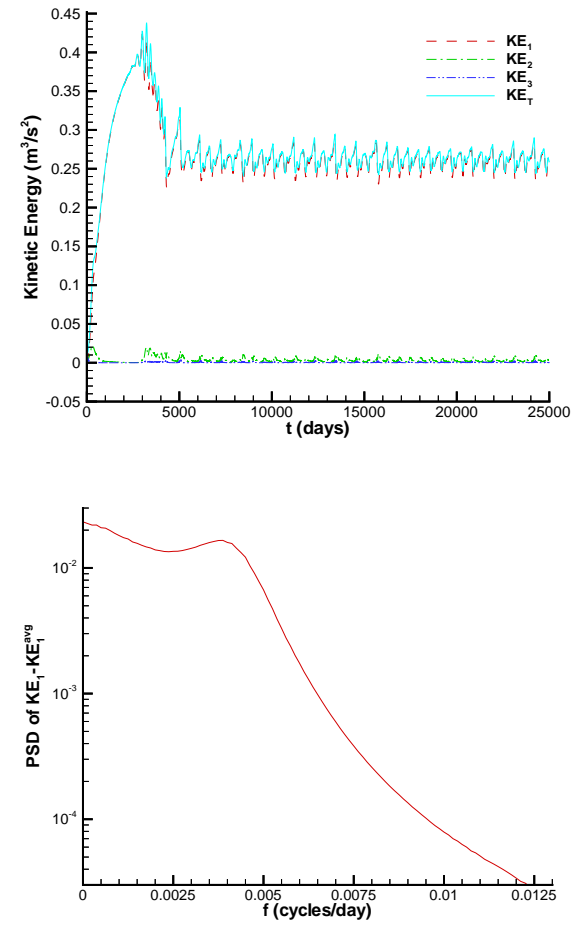

Fig. 19. Spatially-averaged kinetic energy and the corresponding power spectrum for the upper layer when $\tau_{0}=0.1675 \mathrm{dyn} / \mathrm{cm}^{2}$. However, now the spectrum is broadband and not dominated by a small number of frequencies. This situation is typical of chaotic dynamical systems.

present boundary position and shape relative to the initial boundary. This reversible flux value could change significantly as the boundary fluctuated. The variation for northern lobes shown in Fig. 18 is $\phi_{v}=24.2 \%$, which is a significant and non-proportionate increase. This significant increase in the lobe variation is expected since this quasiperiodic case represents a transition to a chaotic flow.

\section{$5.5 \tau_{0}=0.1675:$ A time-chaotic velocity field}

For this value of $\tau_{0}$ the velocity field is temporally chaotic, as can be seen from the broadband spectrum in the lower panel of Fig. 19.

In Fig. 20 we show the lobes created by the intersection of the stable and unstable manifolds of the hyperbolic trajectories on the eastern and western boundaries for $\tau_{0}=0.1675$ and $t_{n}=26600$ days. There are 14 intersection points, all of which are pips. The manifolds and pips define 13 lobes, 6 of which are purple and 7 of which are green. All of the purple lobes indicate fluid which has recently been transported across the boundary from the southern gyre to the northern gyre. Of the green lobes, one of them in the northern gyre along the western boundary indicates fluid which will be transported across the boundary, while 6 of them in the southern gyre indicate fluid which has been recently trans- 


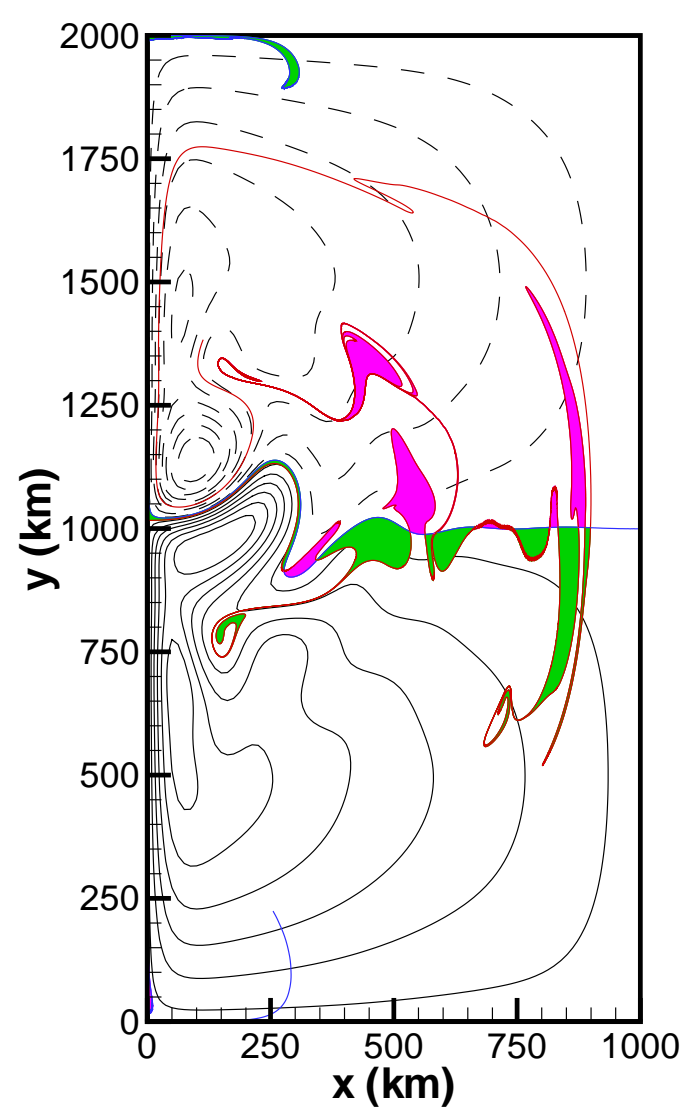

Fig. 20. Lobes created by the intersection of the stable and unstable manifolds of the hyperbolic trajectories on the eastern and western boundaries, respectively, for $\tau_{0}=0.1675 \mathrm{dyn} / \mathrm{cm}^{2}$ at $t_{n}=26600$ days. As in Fig. 15, the lobes have been colorized with alternating green and purple.

ported across the boundary. From these lobes, we can compute the usual transport quantities that we have in previous sections: the intergyre transport is $\phi_{S, N}=0.090 \mathrm{~Sv}$ and the flux along the jet is $\phi_{J}=7.12 \mathrm{~Sv}$. So, in the transition to chaotic flow, there has been a steep decrease both in intergyre transport, as well as flux along the jet. The reversible flux in Fig. 20 is $\phi_{R}=-0.00106 \mathrm{~Sv}$, which represents the first so far where the boundary fluctuation is such that the present boundary at $t=t_{n}$ is on the average, south of the original boundary at $t=t_{0}$.

From Fig. 20, we see that the geometric pattern of the lobes is much more irregular than in the periodic or quasiperiodic cases. In fact, the variation in lobe areas is almost $100 \%$, a dramatic increase over the variation at lower values of $\tau_{0}$. We also note that some of the lobes have formed rings, while others have not. One of the purple lobes in the northern gyre has pinched off to form a ring, and two of the lobes in the southern gyre have also pinched off to form rings.

Fig. 21 shows several steps in the formation of the eastern-most ring in Fig. 20 several months earlier, at $t=$
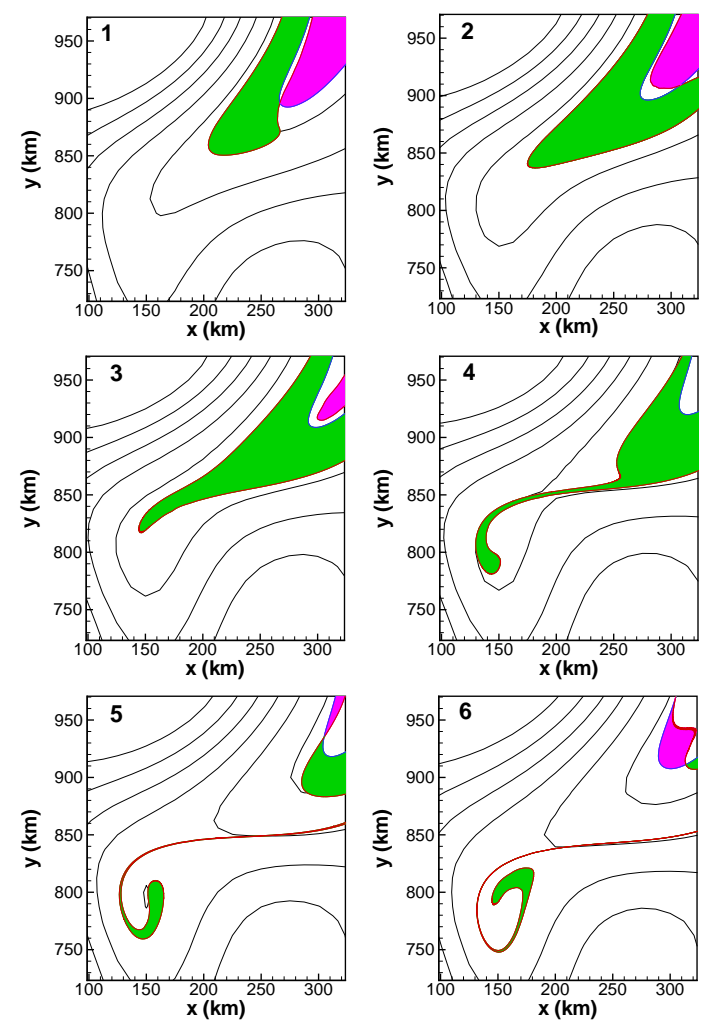

Fig. 21. A series of snapshots showing the formation of a ring in the southern gyre when $\tau_{0}=0.1675$. The upper-left panel starts at $t=25855$ days, and then each following panel depicts the ring formation process at 30 days intervals.

25855 days and spanning a 150 day period, at 30 day intervals. The green lobe represents fluid which has been transported across the jet from the northern gyre. Thus, we can clearly see from this series of snapshots that only the fluid in the exterior of the ring has contributed to intergyre transport and the majority of the fluid in the interior of the ring does not contribute to intergyre transport.

\section{$5.6 \tau_{0}=0.170:$ A time-chaotic velocity field}

Here we have another temporally chaotic case, as is evident from the time series and the spectrum of the spatiallyaveraged kinetic energy shown in Fig. 22.

Despite the different time dependencies, the behavior of the hyperbolic trajectory for chaotic flows is similar to that for periodic flows. In Fig. 23 we show the hyperbolic trajectory as a function of time along the western and eastern boundaries, similar to Fig. 9, but for $\tau_{0}=0.170 \mathrm{dyn} / \mathrm{cm}^{2}$. The convergence of $\alpha_{y}$ and $\beta_{y}$ to the hyperbolic trajectory for both the western and eastern boundary in Fig. 23 is similar to that of Fig. 9, rapid on western boundary, requiring only 34 days to converge to less than $1 \mathrm{~km}$, and much slower for the eastern boundary, again requiring over 2000 days to converge to less than $1 \mathrm{~km}$. As expected, we see that neither the instantaneous vertical position of the stagnation point, nor the 

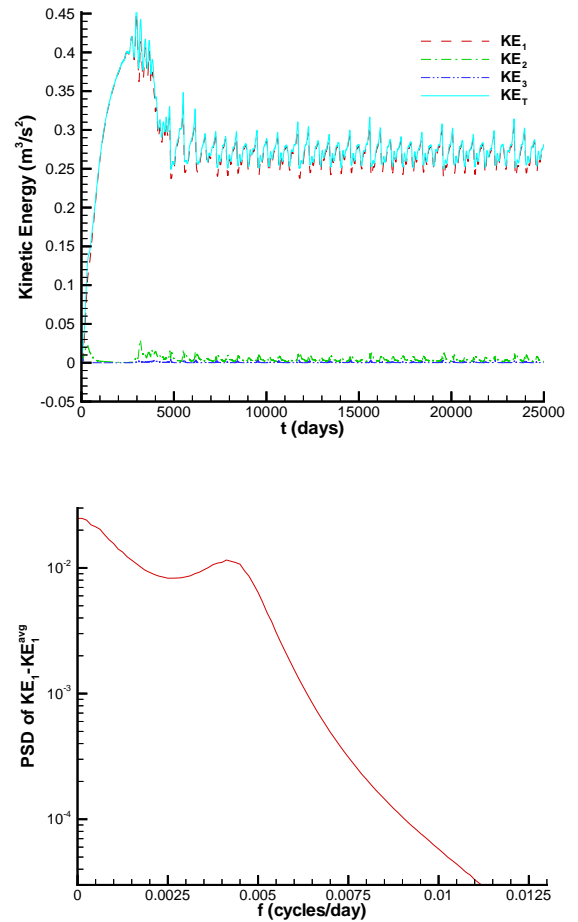

Fig. 22. Spatially-averaged kinetic energy and the corresponding power spectrum for the upper layer when $\tau_{0}=0.170 \mathrm{dyn} / \mathrm{cm}^{2}$. The spectrum shows the same characteristics as in the case shown in Fig. 19.

vertical position of the hyperbolic trajectory follow a sinusoidal path, as they did in Fig. 23. But the behavior of the hyperbolic trajectory relative to that of the stagnation point in both the upper and lower panels of Fig. 23 is similar to that of Fig. 9. In the upper panel, the hyperbolic trajectory follows the stagnation point closely, while in the lower panel, the vertical movement of the stagnation point is much more rapid than that of the hyperbolic trajectory. So, we note that the principal previously discussed: the range of travel of the hyperbolic trajectory is bounded by the range of travel of the stagnation point also holds in this time-chaotic case.

The lobes created by the intersection of the stable and unstable manifolds of the hyperbolic trajectories on the eastern and western boundaries, respectively, for $\tau_{0}=$ $0.170 \mathrm{dyn} / \mathrm{cm}^{2}$ at $t=27925$ days is shown in Fig. 24 . The lobes have again been colorized with alternating green and purple to make them easier to see. There are 16 intersection points, all of which are pips. The manifolds and pips define 15 lobes, 7 of which are purple and 8 of which are green. Of the purple lobes, one of them in the southern gyre along the western boundary indicates fluid that will cross the boundary, while 6 of them in the northern gyre indicate fluid which has recently crossed the boundary. Of the green lobes, 2 of them in the northern gyre along the western boundary are difficult to see, because they are very thin,
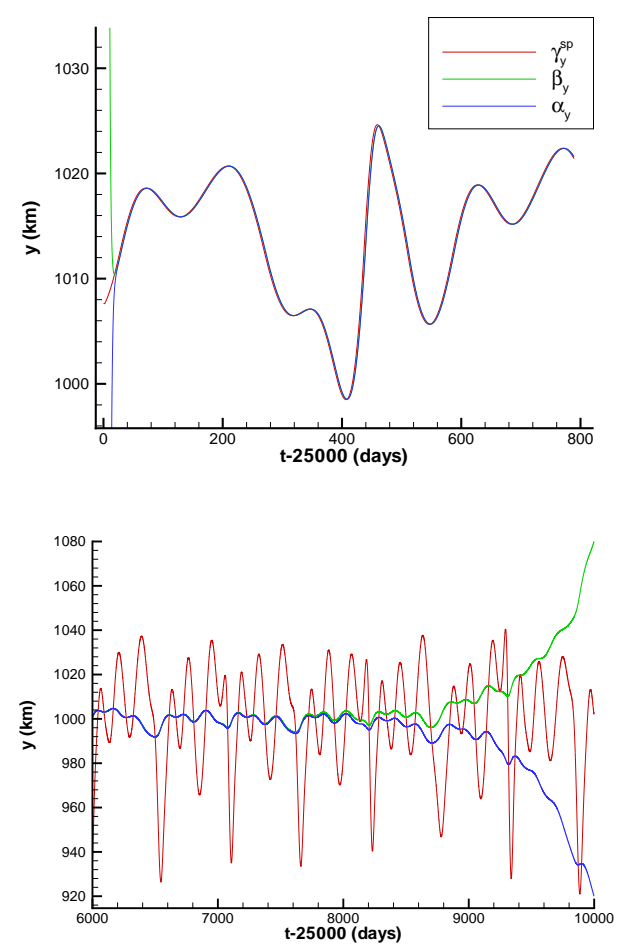

Fig. 23. The location of the hyperbolic trajectory and the instantaneous location of the stagnation point for the western boundary (upper panel) and the eastern boundary (lower panel) when $\tau_{0}=0.170 \mathrm{dyn} / \mathrm{cm}^{2}$.

but still indicate fluid which will cross the boundary into the southern gyre. The remaining 6 green lobes are in the southern gyre and indicate fluid which has recently crossed the boundary from the northern gyre. Note that the lobes vary significantly in size and shape, and in fact, the variation in area of the northern lobes is again nearly $100 \%$. The intergyre flux is $\phi_{S, N}=0.195 \mathrm{~Sv}$ and the flux along the jet is $\phi_{J}=7.158 \mathrm{~Sv}$. So, after the drop in transport that was experienced in the transition from periodic to chaotic time dependence, we see that both $\phi_{S, N}$ and $\phi_{J}$ continue to increase as $\tau_{0}$ is increased in the chaotic regime. As in the last case, the instantaneous value of the reversible flux happens to be $\phi_{R}=-0.0594 \mathrm{~Sv}$, which again indicated that the present boundary is, on the average, south of the original boundary at $t=t_{0}$. We also observe from Fig. 24 that there happen to be two rings present at $t=27925$ days, one in the southern gyre which was formed some time ago, and another in the northern gyre which is just finishing its pinching or formation process. Note that this ring, which is located in the northern gyre, directly above the eastern end of the jet, is significantly different in character than the other rings that we have looked at and discussed so far. The formation of this ring over a 30 day interval is shown in Fig. 25. Note that when this ring is formed, the interior of it is entirely purple, indicating that all of the fluid contained by the ring has contributed to in- 


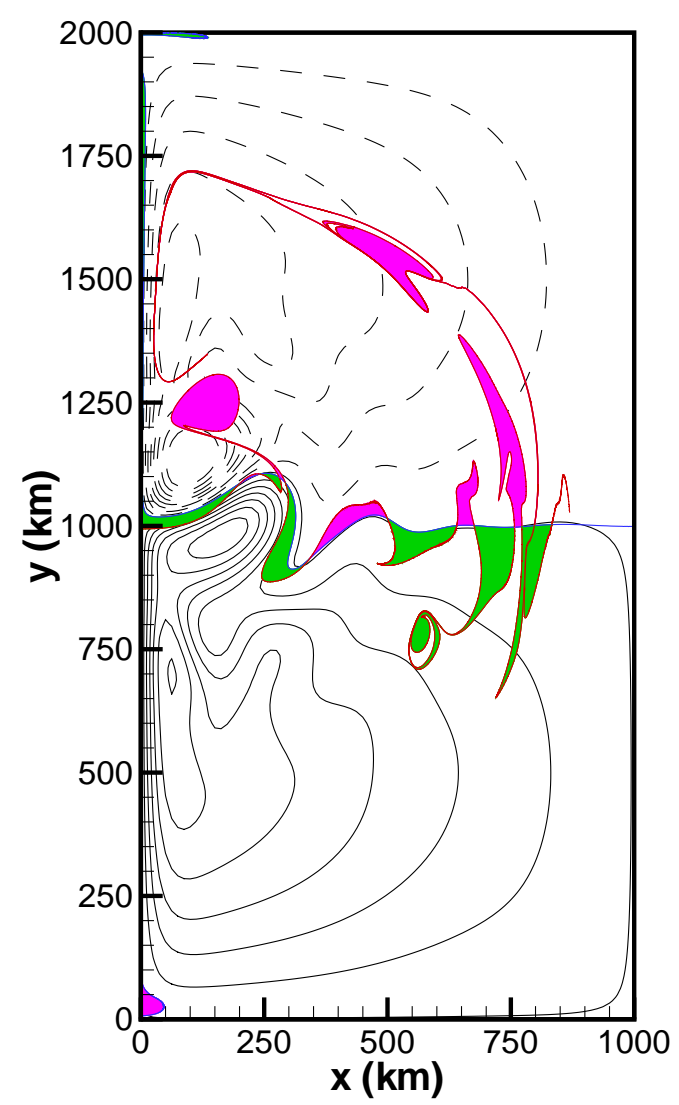

Fig. 24. Lobes created by the intersection of the stable and unstable manifolds of the hyperbolic trajectories on the eastern and western boundaries, respectively, for $\tau_{0}=0.170 \mathrm{dyn} / \mathrm{cm}^{2}$ at $t=27925$ days. As in Fig. 15, the lobes have been colorized with alternating green and purple.

tergyre transport. So, the contribution of rings to intergyre transport can vary tremendously, depending on the time dependence of the flow. In a perfectly time-periodic flow, the rings contribute almost nothing to the intergyre transport and in time-chaotic flows, their entire contents can contribute to intergyre transport.

In Fig. 26 we present results similar to that of Fig. 11 for the reversible and irreversible intergyre flux. Identical to the methods used for Fig. 11, the flux $\phi_{S, N}$ shown in the left panel of Fig. 26, is computed from lobes which transported fluid from the southern gyre into the northern gyre (purple dashed line) and the flux $\phi_{N, S}$ is computed from the lobes which transported from the northern gyre into the southern gyre (green dashed line). Their time averages $\bar{\phi}_{S, N}$ and $\bar{\phi}_{N, S}$ are shown by the solid purple and green lines, respectively. The black and red lines which appear nearly as a single line are the four flux quantities shown in Fig. 11, provided in this figure for comparison purposes. Although the wind stress curl for these two cases presented in Fig. 26 differs only by $0.03 \mathrm{dyn} / \mathrm{cm}^{2}$, there is a striking difference in the behavior of the intergyre flux. First, the magnitude of the flux for the $\tau=0.170$ case is typically more than twice that of the flux
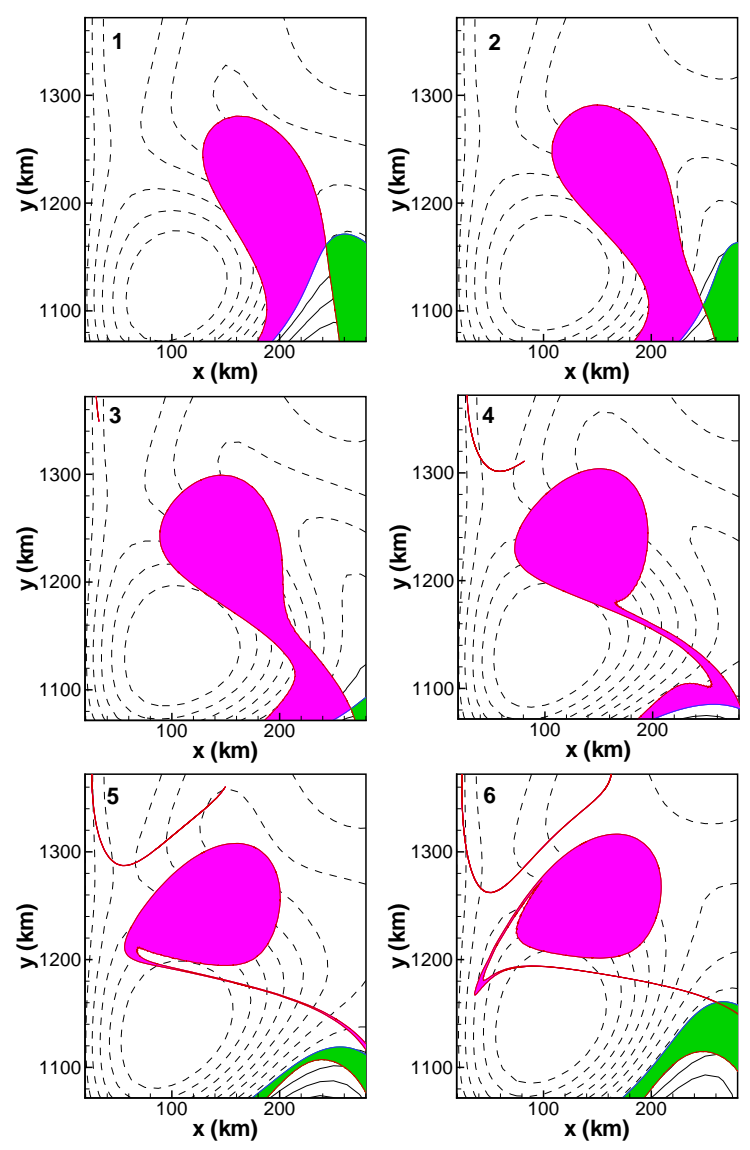

Fig. 25. A series of snapshots showing the formation of a ring in the northern gyre when $\tau_{0}=0.170 \mathrm{dyn} / \mathrm{cm}^{2}$. The upper-left panel starts at $t=27781$ days, and then each following panel depicts the ring formation process at 30 days intervals.

for the $\tau=0.140$ case. Second, the fluctuations in $\phi_{S, N}$ and $\phi_{N, S}$, indicated by the purple and green dashed lines, are several orders of magnitude larger than the fluctuation indicated by the red and black dashed lines. Third, the amount of reversible flux, $\phi_{R}=\phi_{S, N}-\phi_{N, S}$, shown in the right panel of Fig. 26 has considerably larger variations than that shown in the lower panel of Fig. 11. Note that the units of the vertical axis of the lower panel of Fig. 11 are in $\mathrm{m}^{3} / \mathrm{s}$ and the units of the vertical axis of Fig. 26 are in Sv, so the reversible flux in the $\tau_{0}=0.170$ case has variations which are approximately three orders of magnitude larger than the $\tau_{0}=0.140$ case. Also note that $\phi_{S, N}, \phi_{N, S}$ and $\phi_{R}$ have smooth continuous changes over short time segments, and then sudden discontinuous changes, which are a direct result of re-labeling a new pip as the bip. This is necessary since we are maintaining only finite segments of infinitely long manifolds, and therefore only having finite knowledge of the present transport processes. The time averages smooth out the discontinuities due to the changing bip, but it becomes smoother and smoother as the time average becomes longer. Therefore, the time average is useful for obtaining a steady or quasisteady value for the intergyre flux, but not useful for understand- 

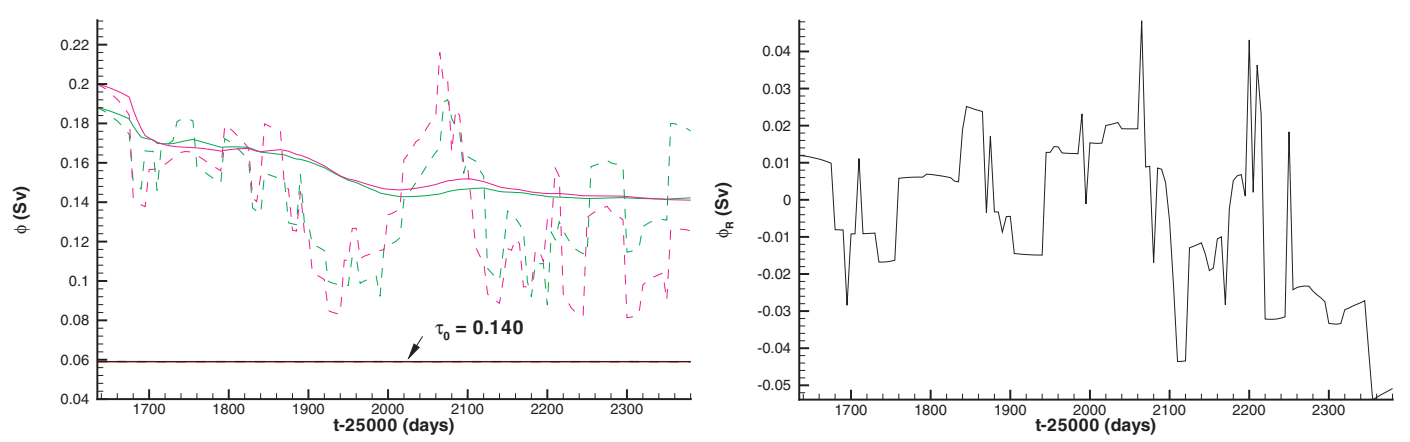

Fig. 26. Flux as a function of time where $\tau_{0}=0.170 \mathrm{dyn} / \mathrm{cm}^{2}$. Similar to Fig. 11, the flux $\phi_{S, N}$ is computed from lobes which transported fluid from the southern gyre into the northern (purple dashed line) and the flux $\phi_{N, S}$ is computed from the lobes which transported from the northern gyre into the southern (green dashed line). Their time averages $\bar{\phi}_{S, N}$ and $\bar{\phi}_{N, S}$ are shown by the solid purple and green lines, respectively. The black and red lines which appear as nearly a single line, are the four flux quantities shown in Fig. 11.
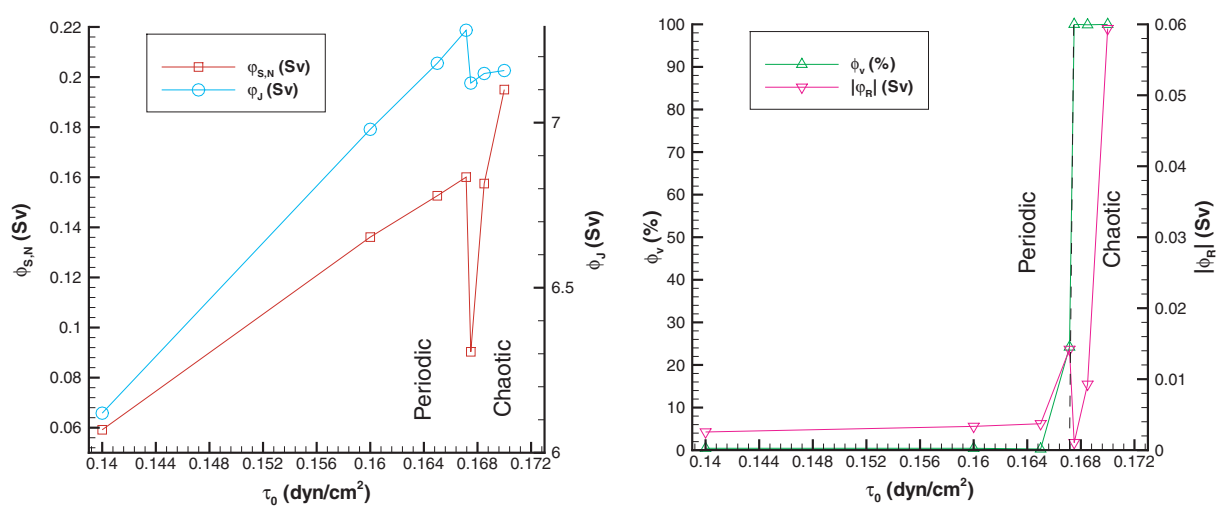

Fig. 27. Summary of the flux computations discussed in the previous sections. $\phi_{S, N}, \phi_{J},\left|\phi_{R}\right|$, and $\phi_{v}$ are each presented as a function of $\tau_{0}$.

ing the present or transient intergyre flux. A better method for computing the flux would be to maintain an average over certain time intervals or a particular number of lobes. However, this is a challenging task, and is thus beyond the scope of this paper. Coulliette et al. (2000) further discuss the details in the computational methods necessary for Fig. 11 and Fig. 26, and those necessary to maintain an average flux over a certain time interval or particular number of lobes.

\subsection{Summary of the flux results}

We summarize our flux results in Fig. 27. The left panel in the figure shows the values of intergyre transport $\left(\phi_{S, N}\right)$ on the left-hand vertical axis as a function of $\tau_{0}$ and values of the transport along the jet $\left(\phi_{J}\right)$ on the right-hand vertical axis as a function of $\tau_{0}$. For both types of transport we see that the flux increases linearly with respect $\tau_{0}$ in the periodic regime, a catastrophic change in the flux-wind relationship occurs in the transition from periodic to chaotic time dependence, resulting in a decrease in flux.
In the right panel of the figure we show the reversible flux and the lobe variation as a function of $\tau_{0}$. Note that the variation is very small, always less than $1 \%$ for the periodic flows, then rises to $24 \%$ for the quasiperiodic case, and then reaches nearly $100 \%$ for each of the chaotic cases. The reversible flux does not follow this type of pattern simply because it is the instantaneous value of the reversible flux at each of the times discussed in the previous sections which represents the present position of the boundary relative to that of the original boundary.

\section{Conclusions}

The most typical approach used to try to understand Lagrangian transport is to release drifters or particles from various locations throughout the flow (e.g. Lozier and Riser, 1989). The result is a collection of particle pathways that are often referred to as "spaghetti plots". Very little useful information can be extracted from these plots, other than the ob- 
servation that Lagrangian transport is difficult to understand. The problem is the nonlinearities inherent in Eq. (5) cause seemingly straightforward velocity fields to yield seemingly unintelligible Lagrangian trajectories. Rather than releasing particles from randomly chosen locations, the dynamical systems approach releases a series of particles from specific locations (hyperbolic trajectories), which identifies two material surfaces (unstable and stable manifold) that intersect each other (pips and sips) and divide the basin into qualitatively different Lagrangian regions (lobes). The visualization of these regions allows us to understand, for example, why a particle released from a specific location at a given time follows a completely different path than a particle released at exactly the same location at a slightly later time. In addition, these Lagrangian regions can be used to compute the precise amount of flux crossing a moving fluid boundary and to visualize the Lagrangian shape of the regions both before and after crossing the fluid boundary. This is not possible with any other conventional method. More specifically we can make the following conclusions regarding Lagrangian transport and intergyre flux:

- It is possible to quantify Lagrangian transport for periodic, quasiperiodic and chaotic time dependence using lobe dynamics with numerically generated velocity fields.

- The intergyre flux is proportional to the flow rate of the jet.

- The intergyre flux and the flow rate of the jet are linear functions of the wind magnitude for periodic flows.

- A catastrophic change occurs in the flux-wind relationship during the transition from periodic to chaotic time dependence.

- The intergyre flux and the flow rate of the jet are nonlinear functions of the wind magnitude for chaotic flows.

Concerning flow structures and the use of invariant manifolds and lobes in describing their influence on transport, we can make the following conclusions:

- It is possible to precisely study the geometric structure of transported fluid using lobe dynamics with numerically generated velocity fields.

- Ring formation from a meandering jet is evident in periodic, quasiperiodic and chaotic flows.

- Ring formation does not contribute significantly to intergyre flux in periodic flows.

- Ring formation does contribute to intergyre flux in chaotic flows.

- All of the fluid that crosses the jet must pass through the western boundary current.
- Fluid that makes a transition around both gyres must follow the "figure eight" pattern dictated by the lobes.

Acknowledgements. We are grateful to Clark David Rowley for use of his $n$-layer quasigeostrophic model (QGN). We thank Kayo Ide for her many insights throughout this effort and Pavel Berloff and James McWilliams for their cooperation in the early phases of this research. We also thank Byron Ponten and David Ossorio of Amtec Engineering (http://www.amtec.com) for their expertise in extending Tecplot with the Lobe Dynamics Add-on. (note: QGN and the Lobe Dynamics Add-on are freeware, available from our web site at http://transport.caltech.edu).

\section{References}

Aref, H. and El Naschie, M. S. (eds.), Chaos Applied to Fluid Mixing, Chaos, Solitons, and Fractals, 4 (6), 1-380, 1994.

Babiano, A., Provenzale, A., and Vulpiani, A. (eds.), Chaotic Advection, Tracer Dynamics, and Turbulent Dispersion, Proceedings of the NATO Advanced Research Workshop and EGS Topical Workshop on Chaotic Advection, Conference Centre Sereno di Gavo, Italy, 24-28 May 1993, Physica D 76, 1-329, 1994.

Coulliette, C., Hatfield, J. and Wiggins, S., Dynamical Systems Analysis of Vector Fields defined as Data Files: Application to Lagrangian Transport and Chaotic Advection, in progress, 2000.

Cummins, P. F. and Mysak, L. A., A quasi-geostrophic circulation model of the northeast Pacific, Part I: A preliminary numerical experiment, J. Phys. Oceanogr., 18, 1261-1286, 1988.

Cushman-Roisin, B., Introduction to Geophysical Fluid Dynamics, Prentice-Hall, Eaglewood, 1994.

del-Castillo-Negrete, D. and Morrison, P. J., Chaotic transport of Rossby waves in shear flow, Phys. Fluids A, 5 (4), 948-965, 1993.

Dijkstra, H. A. and Katsman, C. A., Temporal variability of the wind-driven quasi-geostrophic double gyre ocean circulation: basic bifurcation diagrams, Geophys. Astrophys. Fluid Dynamics 85, 195-232, 1997.

Duan, J. and Wiggins, S., Fluid exchange across a meandering jet with quasiperiodic variability, J. Phys. Oceanogr., 26 (7), 11761188, 1996.

Duan, J. and Wiggins, S., Lagrangian transport and chaos in the near wake of the flow around an obstacle: a numerical implementation of lobe dynamics, Nonlinear Processes in Geophysics, 4, 125136, 1997.

Haller, G. and Poje, A., Finite time transport in aperiodic flows, Physica D, 119(3-4), 352-380, 1998.

Holland, W. R., The role of mesoscale eddies in the general circulation of the ocean-numerical experiments using a wind-driven quasi-geostrophic model, J. Phys. Oceanogr., 8, 363-392, 1978.

Ide, K. and Wiggins, S., Distinguished Hyperbolic Trajectories in Time Dependent Fluid Flows: Analytical and Computational Approach for Velocity Fields Defined as Data Sets, in progress, 2000 .

Jiang, S., Jin, F.-I., and Ghil, M., Multiple equilibria, periodic, and aperiodic solutions in a wind-driven, double-gyre, shallow-water model, J. Phys. Oceanogr., 24, 764-786, 1995.

Lozier, M. S. and Riser, S. C., Potential vorticity sources and sinks in a quasi-geostrophic ocean: beyond western boundary currents, J. Phys. Oceanogr., 20, 1608-1627, 1989. 
Lozier, M. S. and Riser, S. C., Potential vorticity dynamics of boundary currents in a quasi-geostrophic ocean, J. Phys. Oceanogr., 19, 1373-1396, 1990.

Lozier, M. S., Pratt, L. J., Rogerson, A., and Miller, P. D., Exchange geoetry revealed by float trajectories in the Gulf stream, J. Phys. Oceanogr., 27, 2327-2341, 1997.

Malhotra, N. and Wiggins, S., Geometric Structures, Lobe Dynamics, and Lagrangian Transport in Flows with Aperiodic Time Dependence, with Applications to Rossby Wave Flow, Journal of Nonlinear Science, 8, 401-456, 1998.

Miller, P. D., Jones, C. K. R. T. J., Rogerson, A., and Pratt, L. J., Quantifying transport in numerically generated velocity fields, Physica D, 110, 105-122, 1997.

Munk, W.H., On the wind-driven ocean circulation, J. Meteor., 7 79-93, 1950.

Ngan, K. and Shepherd, T. G., Chaotic mixing and transport in Rossby-wave critical layers, J. Fluid Mech., 334, 315-351, 1997.

Pedlosky, J., Geophysical Fluid Dynamics, Springer, New York, 1987.

Phillips, N. A., Energy transformations and meridional circulations associated with simple baroclinic waves in a two-level, quasigeostrophic model, Tellus, 6, 273-286, 1954.

Pierrehumbert, R. T., Chaotic mixing of tracer and vorticity by modulated traveling Rossby waves, Geophys. Astrophys. Fluid Dyn.,
58, 285-319, 1991a.

Pierrehumbert, R. T., Large-scale horizontal mixing in planetary atmospheres, Phys. Fluids A, 3 (5), 1250-1260, 1991 b.

Poje, A.C. and Haller, G., Geometry of cross-stream mixing in a double-gyre ocean model, JPO 29 (8), 1649-1665, 1999.

Pozrikidis, C., Boundary integral and singularity methods for linearized viscous flow, Cambridge University Press, Cambridge, UK, 1992.

Rogerson, A., Miller, P. D., Pratt, L. J., and Jones, C. K. R. T., Lagrangian motion and fluid exchange in a barotropic meandering jet, J. Phys. Oceanogr. 10, 2635-2655, 1999.

Rowley, C., A Modeling Study of the North Atlantic Current, Ph. D. thesis, University of Rhode Island, Kingston, RI., 1996.

Samelson, R. M., Fluid exchange across a meandering jet, J. Phys. Oceanogr., 22, 431-440, 1992.

Shariff, K., Pulliam, T. and Ottino, J., A dynamical systems analysis of kinematics in the time-periodic wake of a circular cylinder, in Vortex Dynamics and vortex methods (ed. C. Anderson and C. Greengard), Proc. AMS-SIAM Conf., Lectures in Applied Mathematics, American Mathematical Society, Providence, 1992.

Spivak, M., A Comprehensive Introduction to Differential Geometry, Vol. II, Publish or Perish, Berkeley, 1979.

Wiggins, S., Chaotic Transport in Dynamical Systems, SpringerVerlag, New York, 1992. 